Article

\title{
New Computational Geometry Methods Applied to Solve Complex Problems of Radiative Transfer
}

\author{
Francisco Salguero-Andújar ${ }^{1}$ and Joseph-Maria Cabeza-Lainez ${ }^{2, *}$ (i) \\ 1 School of Engineering, University of Huelva, Campus de El Carmen, 21007 Huelva, Spain; salguero@uhu.es \\ 2 Higher Technical School of Architecture, University of Seville, 41012 Seville, Spain \\ * Correspondence: crowley@us.es; Tel.: +34-696-749344
}

Received: 11 November 2020; Accepted: 4 December 2020; Published: 6 December 2020

check for updates

\begin{abstract}
Diverse problems of radiative transfer remain as yet unsolved due to the difficulties of the calculations involved, especially if the intervening shapes are geometrically complex. The main goal of our investigation in this domain is to convert the equations that were previously derived into a graphical interface based on the projected solid-angle principle. Such a procedure is now feasible by virtue of several widely diffused programs for Algorithms Aided Design (AAD). Accuracy and reliability of the process is controlled in the basic examples by means of subroutines from the analytical software DianaX, developed at an earlier stage by the authors, though mainly oriented to closed cuboidal or curved volumes. With this innovative approach, the often cumbersome calculation procedure of lighting, thermal or even acoustic energy exchange can be simplified and made available for the neophyte, with the undeniable advantage of reduced computer time.
\end{abstract}

Keywords: mathematics applied to lighting and radiative transfer; configuration factors; computational geometry; parametric design; new solutions for equations of geometric optics; numerical computation of quadruple integrals

\section{Introduction}

\subsection{Form Factors}

To our knowledge, one of the main problems in Science as applied to aerospace, solar and industrial design technology has been to determine how the existing physical fields are transformed according to the physiognomy of fixtures or products and in which direction we should orient our developments to seek a more correct transmission of environmental and heat transfer phenomena, or, in other words, in which way can the design of three-dimensional forms be improved to obtain an optimal and coherent distribution of energies that effectively contributes to mitigate global warming and thereby helps resolve climatic issues.

We must outline that radiation in a physical or spatial environment is made manifest through fields of a fundamentally vector nature. Therefore, our first objective would lie in the assessment of these types of fields in an unaltered state. On such issues, there exist the relevant contributions of Yamauchi [1] and Moon [2,3] amongst others in seeking radiative potential. However, successive modifications of the spatial features of the elements entailed possess the capacity to substantially alter the layout of the field. Such geometric alterations contribute to changes in energy distribution that manufacturers and users are heavily demanding, as it is already vital for them to acquire accurate and simple notions regarding the issue.

The problem is not recent. In fact, Lambert's statement of the famous theorem XVI in his treatise Photometry [4] speculated about the amount of rays (flux) that issues from any two equally luminous surfaces onto the adjacent, and established that if the two surfaces $A_{1}$ and $A_{2}$ were equally luminous 
and faced each other in some way, the amount of incident rays from either of the two surfaces on the second is identical. The former is also known as reciprocity theorem and to develop it to some extent is crucial to assess the mathematical reach of the matter that we are discussing.

The energy that leaves surface $A_{1}$ and reaches surface $A_{2}$ will be:

$$
\mathrm{E}_{1} \mathrm{~A}_{1} \mathrm{~F}_{12}
$$

and, reciprocally, the energy that passes from surface $A_{2}$ to $A_{1}$ is:

$$
\mathrm{E}_{2} \mathrm{~A}_{2} \mathrm{~F}_{21}
$$

where $E_{1}$ and $E_{2}$ are the equivalent amounts of energy (in $W / \mathrm{m}^{2}$ ) emitted by surfaces $A_{1}$ and $A_{2} ;$ and $F_{12}$ or $\mathrm{F}_{21}$ are dimensionless entities called "form factors". If we assume, in principle, that there are no inter-reflections or re-emissions from one surface to the reciprocal, and theoretically no other significant sources of radiation have access, by any means, to the boundaries of the problem, all incident flux will be absorbed and the flow of energy $(\mathrm{d} \Phi)$, according to Lambert's theorem, should be zero, that is:

$$
\mathrm{d} \Phi=\mathrm{E}_{1} \mathrm{~A}_{1} \mathrm{~F}_{12}-\mathrm{E}_{2} \mathrm{~A}_{2} \mathrm{~F}_{21}=0 \Leftrightarrow \mathrm{A}_{1} \mathrm{~F}_{12}=\mathrm{A}_{2} \mathrm{~F}_{21} .
$$

To this end, we would consider the surface elements $\mathrm{dA}_{1}$ and $\mathrm{dA}_{2}$. The angles $\theta_{1}$ and $\theta_{2}$ are measured between the line from $\mathrm{dA}_{1}$ to $\mathrm{dA}_{2}$ (direction of propagation) and the normal to each surface; $r$ is the distance from the center of one differential area to the other (Figure 1):

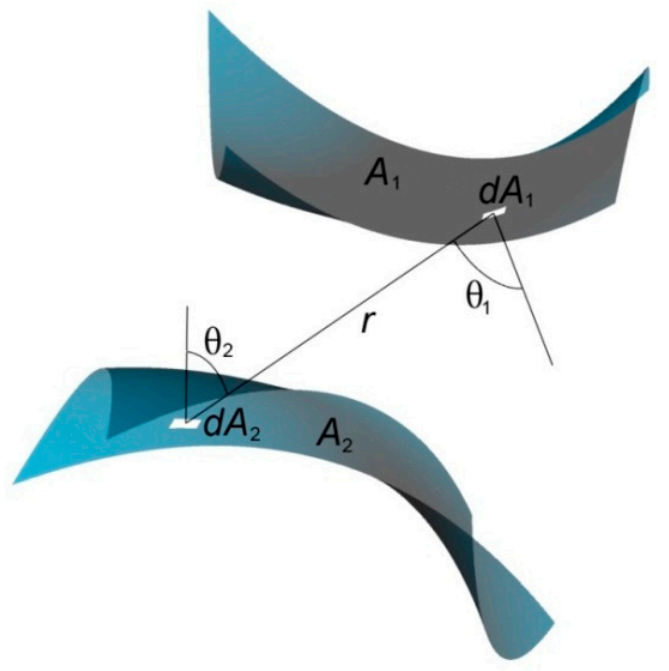

Figure 1. General three-dimensional arrangement of surface source elements through which the radiation form factor is defined.

The cosine emission law, also attributed to J. H. Lambert, states that the flux per unit of solid angle in a given direction $\theta$ (or radiant intensity) is equal to the flux in the normal direction to the surface (maximum intensity) multiplied by the cosine of the angle between the normal and the direction considered.

Luminance or radiance is the radiant flux or power emitted per unit area and solid angle (steradian) in a given direction. To obtain the flow emitted by the surface element $\mathrm{dA}_{1}$ it is necessary to multiply the radiance or luminance by the projected surface in the fixed direction of the angle $\theta_{1}$, and thus:

$$
\mathrm{I}=\frac{\mathrm{d} \Phi}{\mathrm{d} \Omega}=\mathrm{L}_{1} \mathrm{dA} \mathrm{A}_{1} \cos \theta_{1}
$$

where $I$ is the radiant intensity, $d \Omega$ is the solid angle and $L_{1}$ represents the radiance of surface $A_{1}$. 
It is easily deducted that $\mathrm{L}_{1}$ remains independent of the direction and therefore $\mathrm{L}_{1 \theta}=\mathrm{L}_{1}$.

Since the previously found flux refers to the solid angle unit, we must now evaluate the amount of flux that reaches a differential surface element $d A_{n}$ whose distance to $A_{1}$ is precisely $r$.

$$
\mathrm{d} \Phi=\mathrm{L}_{1} \mathrm{dA} \mathrm{A}_{1} \cos \theta_{1} \frac{\mathrm{dA}_{\mathrm{n}}}{\mathrm{r}^{2}}
$$

It is often useful to establish a relationship between luminance or radiance and lighting or irradiance; with this aim, we could simply extend the flux to a hemisphere of radius $r$ in which $\mathrm{dA}_{1}$, is inscribed. The surface $\mathrm{dA}_{\mathrm{n}}$ in spherical co-ordinates amounts to:

$$
\mathrm{dA}_{\mathrm{n}}=\mathrm{r}^{2} \sin \theta_{1} \mathrm{~d} \theta \mathrm{d} \varphi
$$

then from (5) and (6):

$$
\Phi_{1}=\mathrm{L}_{1} \mathrm{~A}_{1} \int_{\mathrm{A}_{\mathrm{n}}} \cos \theta_{1} \frac{\mathrm{dA} \mathrm{A}_{\mathrm{n}}}{\mathrm{r}^{2}}=\mathrm{L}_{1} \mathrm{~A}_{1} \int_{0}^{2 \pi} \int_{0}^{\pi / 2} \cos \theta_{1} \sin \theta_{1} \mathrm{~d} \theta \mathrm{d} \varphi=\pi \mathrm{L}_{1} \mathrm{~A}_{1}=\mathrm{E}_{1} \mathrm{~A}_{1}
$$

Thus, $\mathrm{E}_{1}=\pi \mathrm{L}_{1}$ (E is emitted radiation per area unit, expressed in $\mathrm{W} / \mathrm{m}^{2}$ ).

Returning to the problem of energy exchange, if we assimilate the differential surface element $\mathrm{dA}_{\mathrm{n}}$ with $\mathrm{dA}_{2}$, we find that:

$$
\mathrm{dA}_{\mathrm{n}}=\cos \theta_{2} \mathrm{dA} \text {. }
$$

In this manner, the flux or radiant power that goes from $\mathrm{dA}_{1}$ to $\mathrm{dA}_{2}$, substituting $\mathrm{L}_{1}$ in Equation (5), will be:

$$
\mathrm{d} \Phi_{1-2}=\mathrm{E}_{1} \cos \theta_{1} \cos \theta_{2} \frac{\mathrm{dA}_{1} \mathrm{dA}_{2}}{\pi \mathrm{r}^{2}}
$$

And respectively,

$$
\mathrm{d} \Phi_{2-1}=\mathrm{E}_{2} \cos \theta_{2} \cos \theta_{1} \frac{\mathrm{dA}_{2} \mathrm{dA}_{1}}{\pi \mathrm{r}^{2}} .
$$

The energy exchange is eventually set at:

$$
\Phi_{1-2}=\left(E_{1}-E_{2}\right) \int_{\mathrm{A}_{2}} \int_{\mathrm{A}_{1}} \cos \theta_{1} \cos \theta_{2} \frac{\mathrm{dA}_{1} \mathrm{dA}_{2}}{\pi \mathrm{r}^{2}} .
$$

The above integral equation responds to the fundamental or canonical formula of radiation which in differential terms is:

$$
\mathrm{d}^{2} \Phi=\mathrm{E}_{\mathrm{i}} \cos \theta_{1} \cos \theta_{2} \frac{\mathrm{dA}_{1} \mathrm{dA}_{2}}{\pi \mathrm{r}^{2}}
$$

In such a situation, the integral found by virtue of the fundamental expression adopts the value of $\mathrm{A}_{1} \mathrm{~F}_{12}$ or $\mathrm{A}_{2} \mathrm{~F}_{21}$, following the above definitions. In general, these are quadruple integrals and to solve them becomes so complex that extreme dexterity in geometry problems along with haphazard techniques of integration is required. Therefore, we want to avoid this difficulty as much as possible by virtue of the procedure presented in this article.

The fundamental equation of the much-sought form factors is:

$$
\mathrm{F}_{12}=\frac{1}{\mathrm{~A}_{1}}\left[\int_{\mathrm{A}_{2}} \int_{\mathrm{A}_{1}} \frac{\cos \theta_{1} \cos \theta_{2}}{\pi \mathrm{r}^{2}} \mathrm{dA}_{1} \mathrm{dA}_{2}\right]
$$

and its asymmetric reciprocal in algebraic terms,

$$
\mathrm{F}_{21}=\frac{1}{\mathrm{~A}_{2}}\left[\int_{\mathrm{A}_{2}} \int_{\mathrm{A}_{1}} \frac{\cos \theta_{1} \cos \theta_{2}}{\pi \mathrm{r}^{2}} \mathrm{dA}_{1} \mathrm{dA}_{2}\right]
$$


If we assume, for easier visualization and since it is a frequent case in luminous radiative transfer (but not so in thermal problems involving, for instance. gases), that the surface $\mathrm{A}_{2}$ does not emit energy and only receives it from $A_{1}$, the total flow received in $A_{2}$ will be:

$$
\mathrm{A}_{2} \mathrm{~N}_{2}=\left[\int_{\mathrm{A}_{2}} \int_{\mathrm{A}_{1}} \frac{\mathrm{M}_{1} \cos \theta_{1} \cos \theta_{2}}{\pi \mathrm{r}^{2}} \mathrm{dA}_{1} \mathrm{dA}_{2}\right]
$$

where $M_{1}=N_{2} / F_{21}$ and $N_{2}$ is the energy received by surface 2 in $W / m^{2}$.

\subsection{Configuration Factors}

Let us behold the meaning of the form factor, defined as an integral equation. Actually, what it permits us to calculate is just the net radiant exchange from surface to surface. A surface that emits a certain amount of power $\mathrm{M}_{1}$ (in $\mathrm{W} / \mathrm{m}^{2}$ ) successively produces a certain power $\mathrm{N}_{2}$ 'as an average or percentage' on another surface, which tells us nothing about the point-by-point distribution of that same power.

However, in many engineering, architectural and medical problems, a precise description of the flux function, that is, the radiant field, is often deemed necessary to establish the exact nature of the radiation "map" and to be able to control the possible deficit or surplus which could, in turn, become critical.

Therefore, if we look for the point distribution of power that reaches surface $A_{2}$ instead of the mere average, we could reduce the fourfold integrals in the expression of the form factor to a simpler surface integral as follows:

$$
\mathrm{n}_{2}=\int_{\mathrm{A}_{1}} \frac{\mathrm{M}_{1} \cos \theta_{1} \cos \theta_{2}}{\pi \mathrm{r}^{2}} \mathrm{dA}_{1}=\mathrm{M}_{1} \int_{\mathrm{A}_{1}} \frac{\cos \theta_{1} \cos \theta_{2}}{\pi \mathrm{r}^{2}} \mathrm{dA}_{1}
$$

This new expression is, like the previous one, strictly geometric and as such only depends on angles and areas, given that for a certain moment of time the value of $\mathrm{M}_{1}$ can be assumed as constant.

The double integral equation,

$$
\mathrm{f}_{12}=\int_{\mathrm{A}_{1}} \frac{\cos \theta_{1} \cos \theta_{2}}{\pi \mathrm{r}^{2}} \mathrm{dA}_{1}
$$

will be called the configuration factor and it presents several fundamental applications in the method that we will develop hereby.

The first elementary consequences are:

$$
\mathrm{n}_{2}=\mathrm{f}_{12} \mathrm{M}_{1}
$$

and

$$
\mathrm{A}_{2} \mathrm{~N}_{2}=\mathrm{M}_{1}\left[\int_{\mathrm{A}_{2}} \mathrm{f}_{12} \mathrm{dA}_{2}\right]=\mathrm{A}_{1} \mathrm{~F}_{12} \mathrm{M}_{1}=\mathrm{A}_{2} \mathrm{~F}_{21} \mathrm{M}_{1}
$$

Then,

$$
\mathrm{F}_{12}=\frac{1}{\mathrm{~A}_{1}}\left[\int_{\mathrm{A}_{2}} \mathrm{f}_{12} \mathrm{dA}_{2}\right]
$$

Accordingly,

$$
\mathrm{F}_{21}=\frac{1}{\mathrm{~A}_{2}}\left[\int_{\mathrm{A}_{2}} \mathrm{f}_{12} \mathrm{dA}_{2}\right]
$$

Equation (21) can be clearly identified with the "average" of $\mathrm{f}_{12}$ "over surface" $\mathrm{A}_{2}$, especially since we had already obtained that $\mathrm{N}_{2}=\mathrm{F}_{21} \mathrm{M}_{1}$. In quantum dynamics terms, the form factor expresses the probability of a surface being stricken with photonic energy emitted by an adjacent source. 
Similarly, we would define $\mathrm{N}_{2}$ as the average of $\mathrm{n}_{2}$ on the surface $\mathrm{A}_{2}$;

$$
\mathrm{N}_{2}=\frac{1}{\mathrm{~A}_{2}}\left[\int_{\mathrm{A}_{2}} \mathrm{n}_{2} \mathrm{dA}_{2}\right]
$$

This important finding represents the ignored relationship between form factors and configuration factors that will allow us to easily simplify and visualize many complex calculations in the following chapters by restricting them only to second order primitives, and the ensuing "average" is ready accessible by numerical procedures. To our knowledge, it has only been defined by Cabeza-Lainez $[5,6]$.

\section{Symbolic Calculus of Basic Elements}

\subsection{Triangle}

A set of basic forms can be derived from the triangle, but only if the calculation axis lies perpendicular to the center of the figure or the vertex of the triangle or rectangle considered. The principle equation is,

$$
\mathrm{n}=\mathrm{L} \int_{0}^{\mathrm{b}} \int_{0}^{\mathrm{z}} \frac{\mathrm{y}^{2}}{\left(\mathrm{x}^{2}+\mathrm{y}^{2}+\mathrm{z}^{2}\right)^{2}} \mathrm{dzdx}
$$

for an instant of time $t$ in which the luminance or radiance $\mathrm{L}$ can be considered constant and keeping in mind that $\mathrm{L} \pi=\mathrm{M}$.

The solution for triangle $A_{1}$ in Figure 2, integrated by parts is:

$$
\mathrm{n}=\frac{\mathrm{L}}{2}\left[\frac{\mathrm{b}}{\sqrt{\mathrm{y}^{2}+\mathrm{b}^{2}}} \tan ^{-1} \frac{\mathrm{a}}{\sqrt{\mathrm{y}^{2}+\mathrm{b}^{2}}}\right]
$$

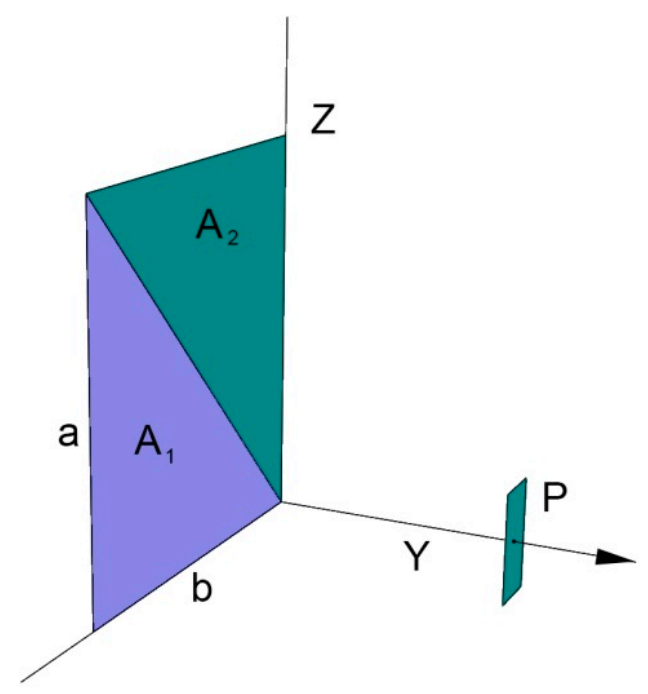

Figure 2. Configuration factor between a rectangle consisting of two united triangles $\left(\mathrm{A}_{1}\right.$ and $\left.\mathrm{A}_{2}\right)$ and a point belonging to a parallel plane.

\subsection{Rectangle}

The full rectangle is just the sum of solutions for surfaces $A_{1}(24)$ and $A_{2}$ (see Appendix $A$ ):

$$
\mathrm{n}=\frac{\mathrm{L}}{2}\left[\frac{\mathrm{a}}{\sqrt{\mathrm{y}^{2}+\mathrm{a}^{2}}} \tan ^{-1} \frac{\mathrm{b}}{\sqrt{\mathrm{y}^{2}+\mathrm{a}^{2}}}+\frac{\mathrm{b}}{\sqrt{\mathrm{y}^{2}+\mathrm{b}^{2}}} \tan ^{-1} \frac{\mathrm{a}}{\sqrt{\mathrm{y}^{2}+\mathrm{b}^{2}}}\right]
$$


However, we need to be reminded that we have just obtained the value of radiative exchange at a single point located precisely under the vertex of the rectangle and in a particular direction (perpendicular) to the surface source.

Deft combinations of triangles would give us the equilateral triangle, hexagon and, as a limit case that we will discuss in Appendix A, the circle, which has been only obtained in such "polygonal" fashion by Cabeza-Lainez.

\subsection{Calculations in a Plane Perpendicular to the Figure}

In the above discussion, we have solely found the component of radiation that was perpendicular to the emitting surface at an isolated point.

It is easy to imagine the severe complexity that arises when trying to find the solution for points moving freely in a plane, let alone space.

Even so, radiance being a vector, to complete the problem means finding values of other auxiliary components.

In this situation, the principle integral turns out to be (if we multiply by the corresponding cosine):

$$
\mathrm{n}=\mathrm{L} \iint \frac{\mathrm{zy}}{\left(\mathrm{x}^{2}+\mathrm{y}^{2}+\mathrm{z}^{2}\right)} \mathrm{dxdz}
$$

Applying this proposition to the case considered (Figure 3), we find that if $Y$ (the distance) is a constant, the integral equation yields:

$$
\mathrm{n}=\frac{\mathrm{L}}{2}\left[\tan ^{-1} \frac{\mathrm{b}}{\mathrm{y}}-\frac{\mathrm{y}}{\sqrt{\mathrm{a}^{2}+\mathrm{y}^{2}}} \tan ^{-1} \frac{\mathrm{b}}{\sqrt{\mathrm{a}^{2}+\mathrm{y}^{2}}}\right]
$$

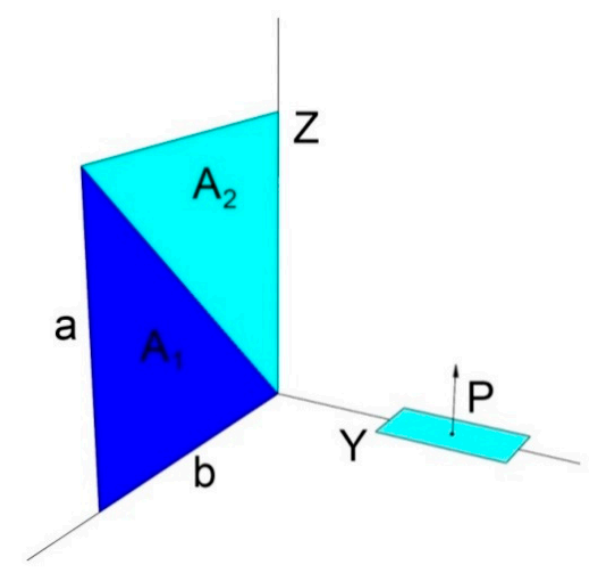

Figure 3. Configuration factor between a rectangle consisting of two annexed triangles $\left(\mathrm{A}_{1}\right.$ and $\left.\mathrm{A}_{2}\right)$ and a point aligned with the normal to a vertex and contained in a plane perpendicular to the figure.

By virtue of the former, we have exposed how complex it becomes to find an analytical expression to solve the problem if only we alter the coordinate plane of calculation, not to mention figures of geometry other than those limited by straight lines. That is why we concluded that there was a significant need for a graphical solution based on parametric design as this would imply a universal solution and an extraordinary simplification of the issue.

\subsection{Calculation of Integral Equation for a 'Rectangle' Over a Horizontal Square}

Previously (Equation (27)) we had found the solution in terms of arctangent for the entire rectangle. The adaptation of this formula to an arbitrary movement of points on a horizontal surface (Figure 4), 
requires taking into account various singularities depending on the position of the rectangle's base, the situations of symmetries and what a unique "form factor algebra" permits.

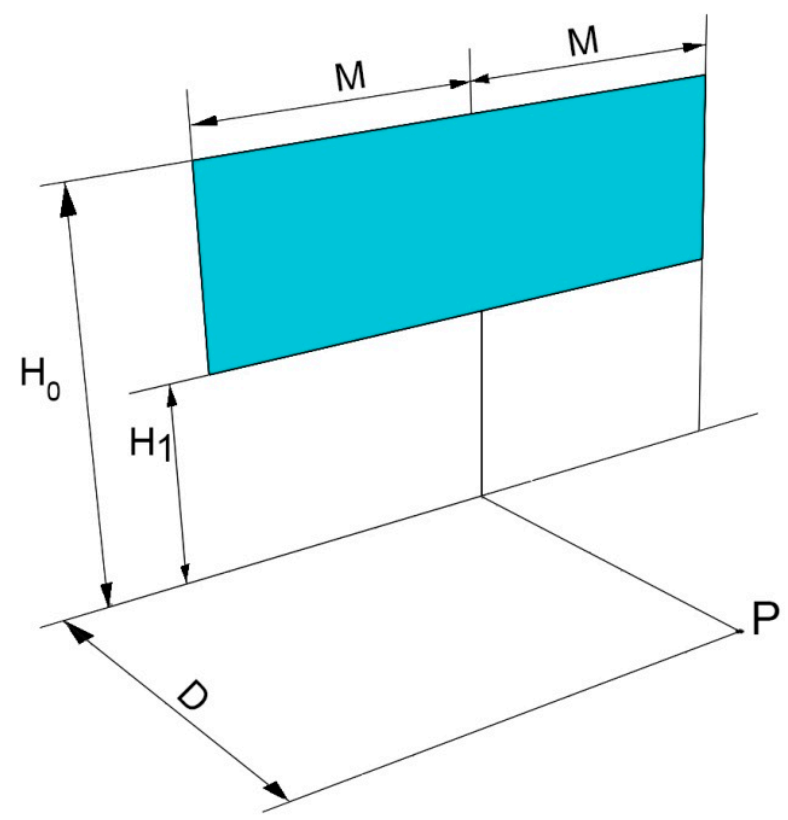

Figure 4. Outline of a 'typical' rectangle to be used for the calculations.

For example, in the $\mathrm{Y}$ axis and at level $-\mathrm{H}_{1}$, the adapted solution would give:

$$
\mathrm{n}=\mathrm{L}\left[\frac{\mathrm{D}}{\sqrt{\mathrm{H}_{1}^{2}+\mathrm{D}^{2}}} \tan ^{-1}\left(\frac{\mathrm{M}}{\sqrt{\mathrm{H}_{1}^{2}+\mathrm{D}^{2}}}\right)-\frac{\mathrm{D}}{\sqrt{\mathrm{H}_{0}^{2}+\mathrm{D}^{2}}} \tan ^{-1}\left(\frac{\mathrm{M}}{\sqrt{\mathrm{H}_{0}^{2}+\mathrm{D}^{2}}}\right)\right]
$$

\section{Methodology}

\subsection{Solid Angle Projection Law}

To obtain the energy exchange between a given surface and a point $\mathrm{P}$, we would simply trace a cone whose vertex is the point considered $P$, and its base is the surface in question $(\mathrm{d} \Omega)$, and proceed to intersect the said cone with a sphere of unit radius (Figure 5). The enclosure within this intersection, projected on any reference plane that we may require and divided by the horizontal area of the aforementioned unit sphere (that is, divided by $\pi$ ), will equate to the value of the configuration factor determined by virtue of the analytical methods of exact integration described in the previous chapters.

If, by means of what we have discussed in detail in the above sections, we were trying to find certain geometric factors or proportions, it seems equally reasonable to achieve these same values through graphic procedures such as the ones employed in Descriptive Geometry.

The advantages are obvious to the project engineer, because if, due to the difficulty of the mathematical problem, hesitation appears, we are allowed to somehow 'visualize' the entire process.

In summary, what has been proven is that the configuration factor is a mere proportion, a dimensionless number, consisting of a ratio of areas, one of them the area of a certain projection and the other the total area involved expressed in circular terms, usually $\pi[6]$.

The most important consequence of the former is that the problem, regardless of its complexity, will always present a unique non-trivial solution since the projection will have a univocal value and cannot be non-existent. 


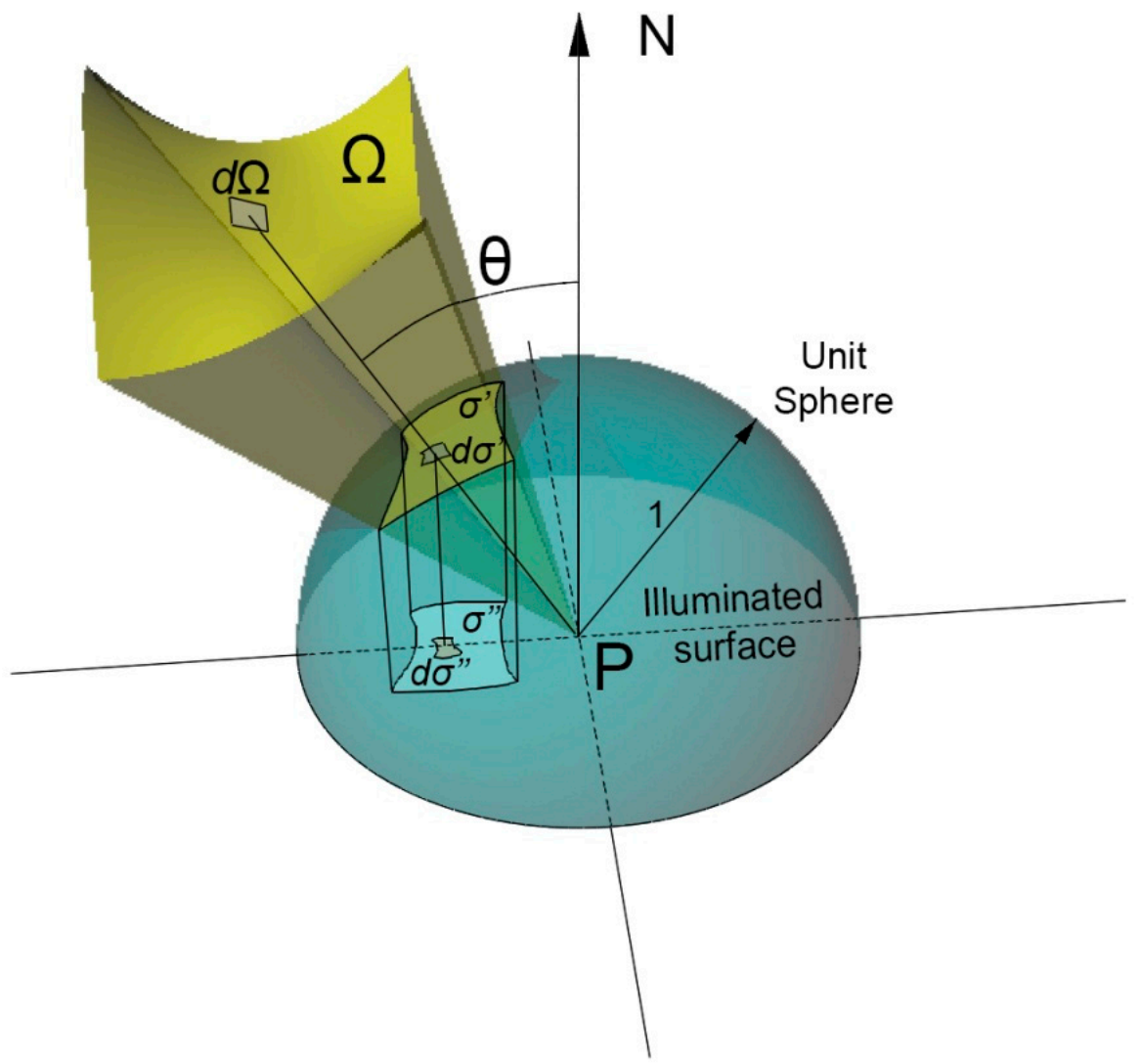

Figure 5. The cone of radiation of this figure cuts the area $\sigma^{\prime}$ on the unit sphere. The orthogonal projection of $\sigma^{\prime}$ over the irradiated plane produces the area $\sigma^{\prime \prime}$. Such area divided by $\pi r^{2}(r=1)$ equates to the configuration factor.

In addition, as the configuration factors are actually projections, they hold an additive property, which is useful to know when dealing with sundry and simultaneous emitting surfaces, because it is possible to add their effects. The average over the receiving surface of the configuration factors thus obtained will constitute the form factor that had been so laboriously sought for by means of mathematical formulation and solving of fourfold integral equations.

Alternatively, graphic methods and analytical methods or a combination thereof can be used. This is the gist of our procedure.

It also seems gratifying to assume that we have solved the fundamental problem of radiation with geometric methods. Why is that? Because it means that 'form' is very important in this type of problem and that not all forms act in the same way from a radiative point of view; some geometries favor exchanges more than others and the mission of the heat transfer analyst is to adequate these forms. predicting their impact. Such repercussion can also be verified by observers, that is, manufacturers, society, etc., using, as we shall see, a simple tool.

However, the sole hindrance that we identified at the time was that numerical validation of the simple rectangle over a horizontal plane (Figure 4) was not readily available, thus casting a shadow over the viability of our approach. Fortunately, Cabeza-Lainez was able to work out an exact solution and this relevant finding is presented in Appendix B.

Then a similar problem arose with circular emitters in a free position. Cabeza-Lainez has completely solved this anew [7], as described in Appendix C, as it may help to validate other curved geometries.

\subsection{Algorithms Aided Design}

Although today, most Computer Aided Design (CAD) programs incorporate what is known as parametric design (which implies that it is not necessary to redraw the objects that we design if 
they change shape, position or size), we should not consider all of these as Algorithm Aided Design programs (AAD).

An algorithm is a logical expression that leads to procedures to be performed through finite sets of closed basic instructions. Algorithms simulate human abilities to break down a problem into a series of simple steps that can be easily executed and, although they are closely related to computers, algorithms can be defined independently of programming languages and applications.

However, not just any procedure can be considered properly as an algorithm, since many procedures are far from being well defined or contain ambiguities, logical contradictions or infinite loops.

Some important properties that are required for a procedure to be considered an algorithm are:

- An algorithm is a well-defined set of properly concise instructions.

- An algorithm demands a defined set of inputs that may or may not come from the output of a previous algorithm.

- An algorithm generates a precise output (Figure 6):

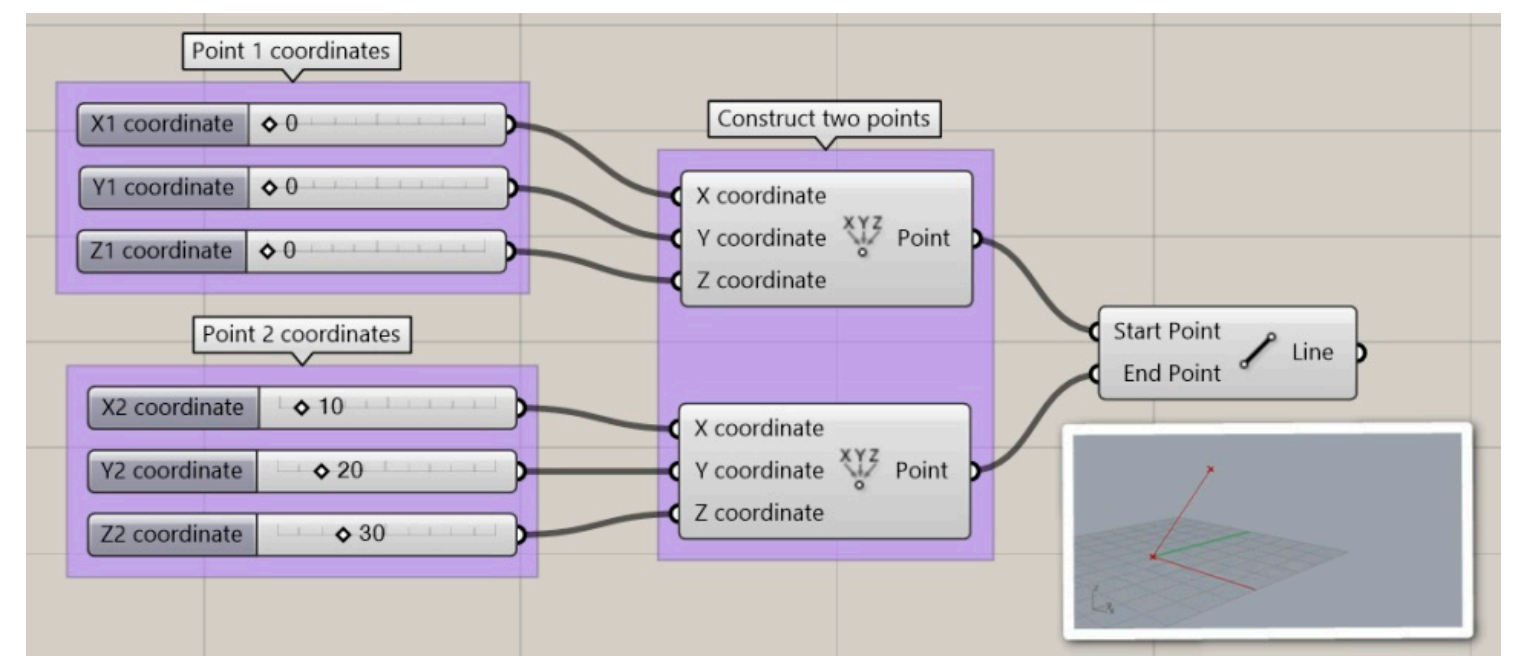

Figure 6. Algorithm programmed in Grasshopper ${ }^{\circledR}$ to obtain all the straight lines.

- Finally, an algorithm can produce warnings and error messages through the appropriate editor. If the inputs are not appropriate, e.g., if we enter text instead of numbers, the algorithm will return an error message instead of the expected output, via the appropriate editor [8].

In addition, through parameterization, we have not merely obtained a straight line, (Figure 6) but, by simply modifying the value of the coordinates, moving the mouse over the sliders we can alter the 'line' output and draw all the straight lines contained in the Euclidean space with a single gesture of the tracer.

\subsection{Calculation by Algorithms Aided Design through Finite Element Method}

The main problem of analytical calculation is that, although we may employ a convenient algebra of form factors [6] to find the radiance received by a surface, issuing from basic figures limited by straight lines, the number of shapes of the emitters, whose direct integration is very difficult or impossible to perform, remains numberless.

To solve such an impasse, we have successfully developed a Grasshopper ${ }^{\circledR}$ algorithm that allows us to calculate the configuration factor subtended by an arbitrary point of any surface (i.e., irrespective of its shape and orientation) from an emitter or 'three-dimensional figure', with manifold geometry or inclination (including those whose integration is deemed impossible). 
To calculate the incident radiation $\mathrm{n}\left(\mathrm{W} / \mathrm{m}^{2}\right)$, we will only have to add up all the configuration factors that we may require and multiply them by the luminance that departs from the emitter $\left(\mathrm{W} / \mathrm{sr} \cdot \mathrm{m}^{2}\right)$.

The steps which we have followed to develop the algorithm can be summarized below:

3.3.1. Geometric Definition of Both the Irradiated Surface and the Emitter and, Subsequently, Division of the Irradiated Surface into a Grid of $\mathrm{N} \times(\mathrm{N}-1)$ Squares, Locating Its Corners

Let us consider for example, a rectangle $2 \mathrm{~m}$ wide by $2 \mathrm{~m}$ high whose base is $5 \mathrm{~m}$ above the ground. The rectangle's vertical axis coincides with the middle point of the horizontal receiving surface of $10 \times 10 \mathrm{~m}$.

First we establish the plane of the irradiated surface defining, by means of sliders, the components of its normal vector and using the 'Vector XYZ' function, constructing the said plane with the help of the 'Plane Normal' function as shown in the upper part of the following figure (Figure 7).

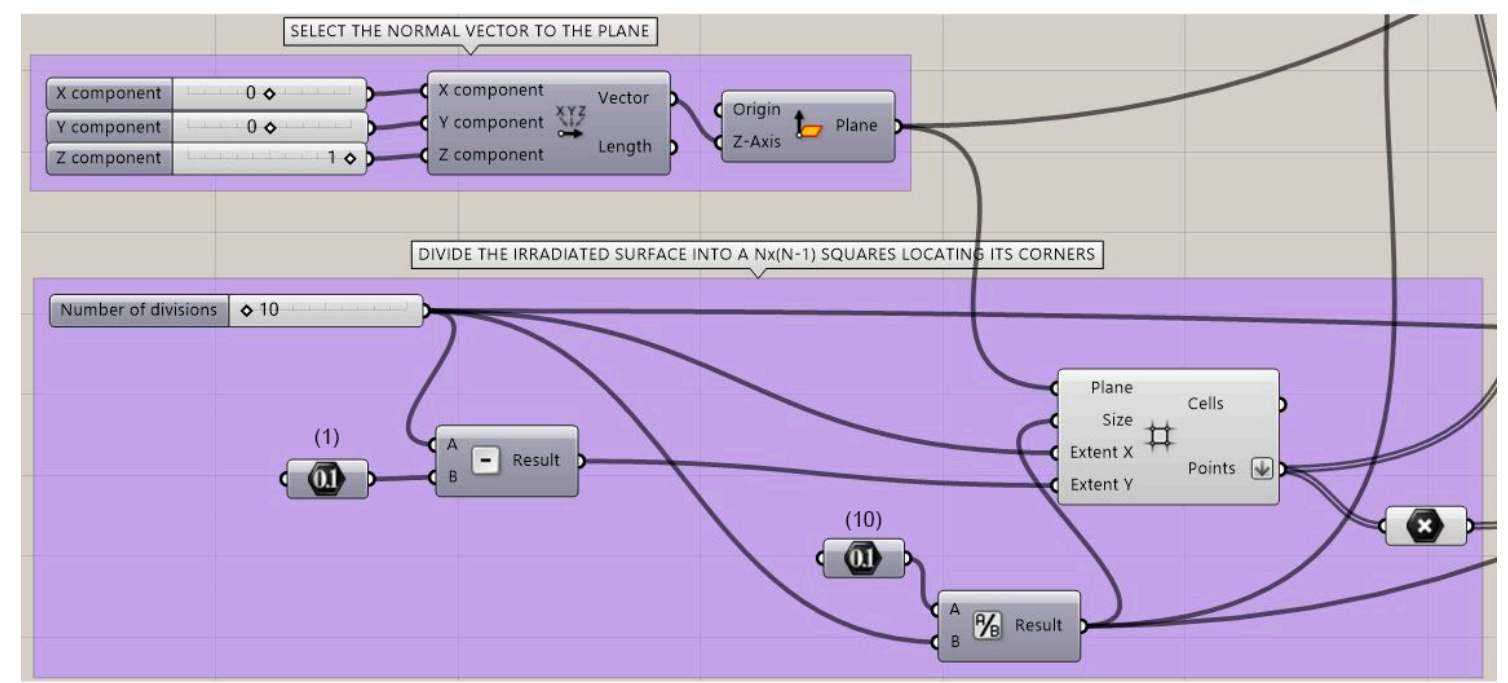

Figure 7. First part of the algorithm.

Subsequently, we divide the irradiated surface into $\mathrm{N} \times(\mathrm{N}-1)$ squares (finite elements) using the 'Square' function that generates a bi-dimensional grid with square cells and retains the points at the corners of the squares.

Note that (in this case) the first row of corner points has been cancelled, since in the plane of the 'rectangle', the radiance will necessarily be equal to zero and the size of the squares is divided by its number for a better performance of the algorithm (Figures 7 and 8):

The result is detailed in Figure 8:

\subsubsection{Calculate the Configuration Factors}

To perform this, it is convenient to select a 'rectangle' (arbitrary), draw the spheres centered in each corner of the divisions (through the 'Sphere' command) and extrude 'light cones' based on the 'rectangle' and vertex positioned at the corners of the divisions ('Extrude point' command).

In the ensuing operation, we have to draw the intersections between the 'light cones' and the spheres, (Boundary representation vs. Boundary Representation: 'Brep|Brep' command), project these intersections on the selected plane ('Project' command) and calculate the areas involved ('Area' command). Finally, we normalize the results to obtain dimensionless configuration factors (Figure 9): 

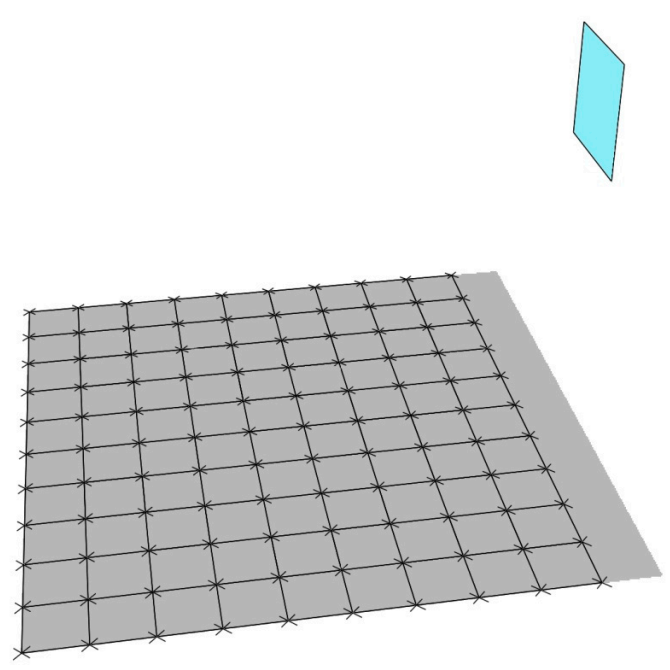

Figure 8. An example of a rectangle $2 \mathrm{~m}$ wide by $2 \mathrm{~m}$ high, and whose base lies $5 \mathrm{~m}$ above the ground. (We assume for convenience that the horizontal surface is a square of $10 \times 10 \mathrm{~m}$ ).

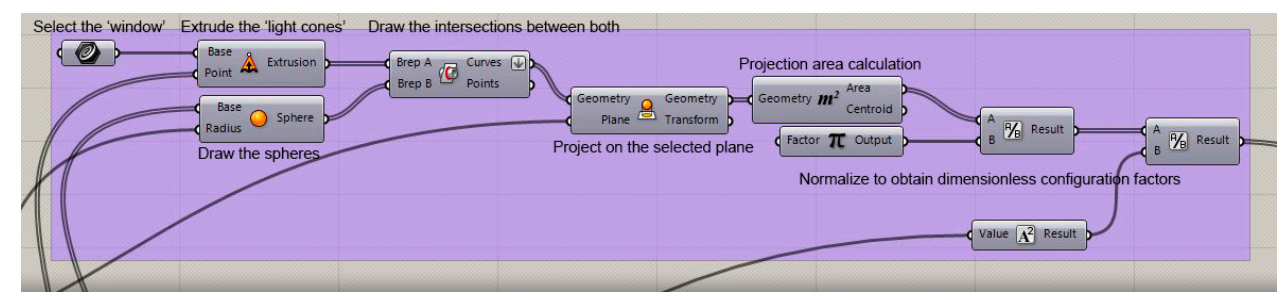

Figure 9. Algorithm to draw $\mathrm{N} \times(\mathrm{N}-1)$ spheres in the corner of the subdivisions.

The result, as expected, is depicted below (Figure 10):

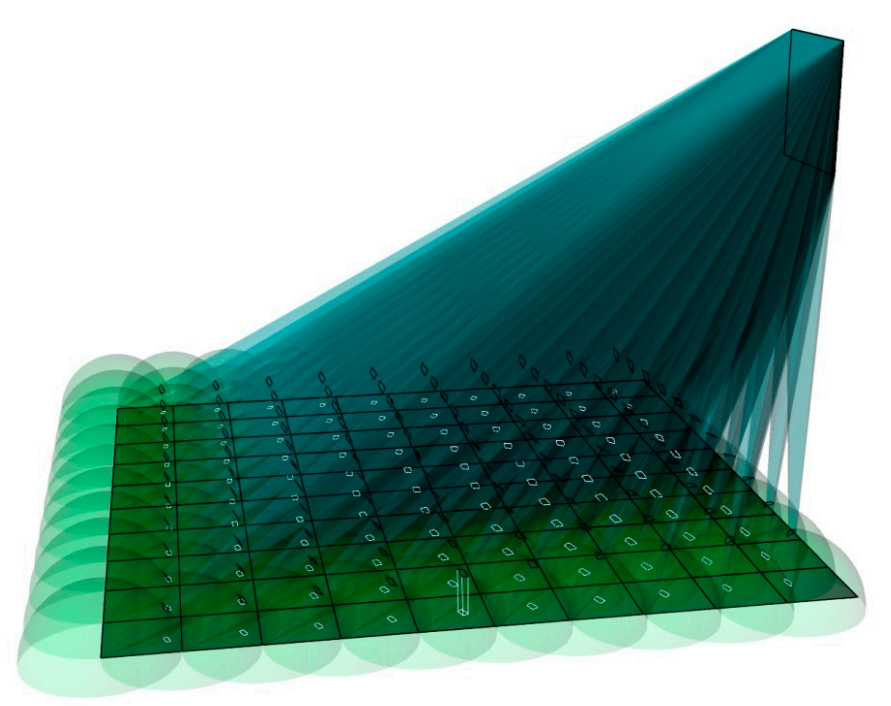

Figure 10. Visualization of the intersections between the 'radiant cones' and the unit spheres and their corresponding projections on the chosen plane.

\subsubsection{Exporting the Data to Excel and Representing them by Color Maps}

To transfer the data to Excel ${ }^{\circledR}$, we first sort them using the 'Partition List' command and then the 'GhExcel' plugin that allows us to export an array of data to the said spreadsheet through the 'ExcelWrite' command, (Figure 11). 


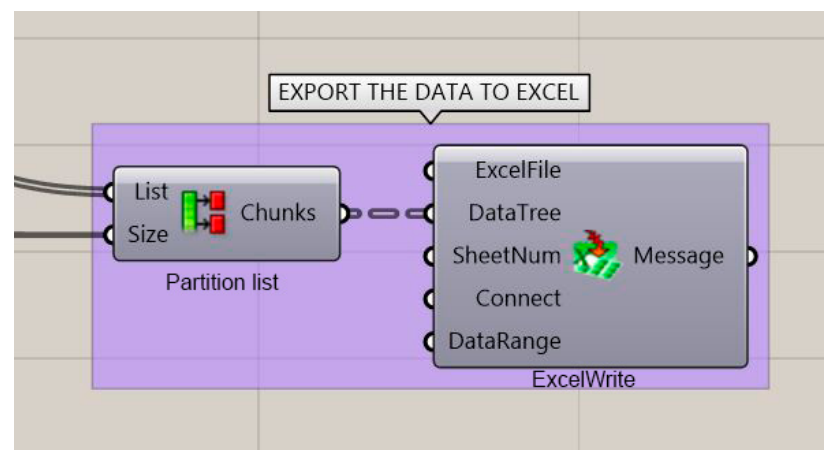

Figure 11. Algorithm to sort and export the data to Excel ${ }^{\circledR}$.

Finally, we have designed a 'renderer' which assigns to each value obtained a color of the spectrum to display the results, (Figure 12):

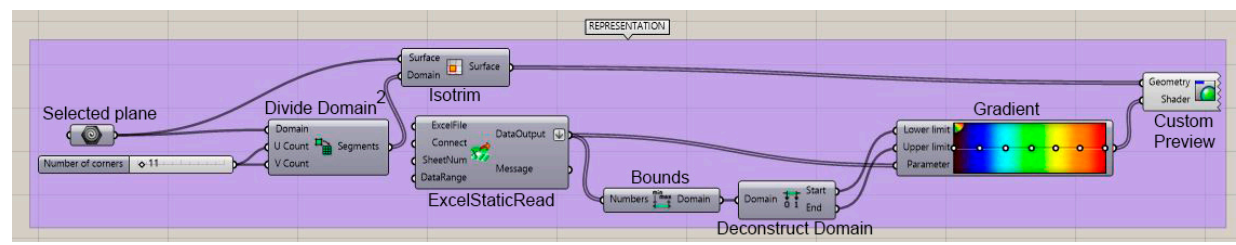

Figure 12. Algorithm designed to graph the results.

The outcome for such an algorithm is shown below (Figure 13).
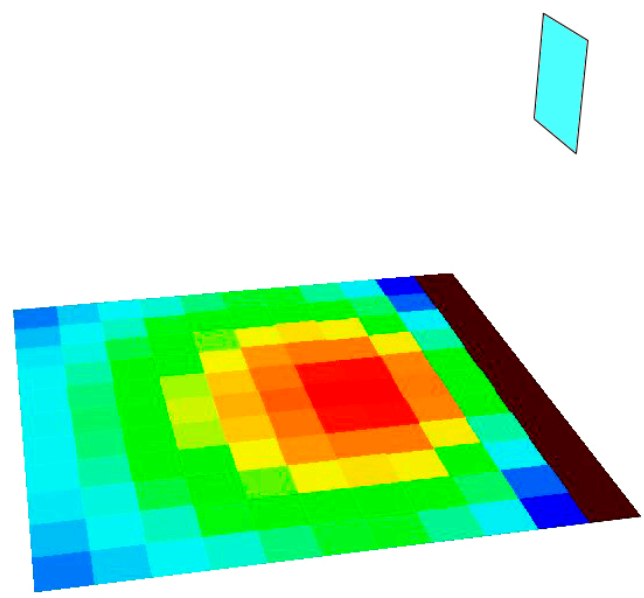

Figure 13. Color map of the configuration factor obtained by the discretization for each meter (110 values) of a horizontal area of $10 \times 10 \mathrm{~m}^{2}$, illuminated by a 'rectangle' of $2 \times 2 \mathrm{~m}^{2}, 5 \mathrm{~m}$ above the ground and whose vertical axis coincides with the middle point of the horizontal surface.

\subsubsection{Numerical Results}

When exporting the values obtained by the Grasshopper ${ }^{\circledR}$ algorithm to an Excel ${ }^{\circledR}$ sheet, it is relatively easy to sort them in a matrix and the figures obtained are displayed in Table 1.

In this manner, the form factor $F_{12}$ is obtained as the average of all the configuration factors $\mathrm{f}_{12}$, which yields a value (for a discretization of $11 \times 11=110$ points) of $F_{12}=0.00556888$.

If we consider the point with the highest configuration factor (point 5,3 ), where $f_{12}=0.01144$, and we want to know the radiation of that point, assuming a radiant power in the rectangle of $1000 \mathrm{~W} / \mathrm{m}^{2}$, we will obtain this by simply multiplying the value of $\mathrm{f}_{12}$ by 1000 , that is, $\mathrm{n}=11.44 \mathrm{~W} / \mathrm{m}^{2}$.

The array presented in Table 1 coincides with that found by the analytical procedures (Table 2) described in the references [6]. In this way the procedure is validated. 
Table 1. Configuration factors of an area of $10 \times 10 \mathrm{~m}^{2}$ illuminated by a 'rectangle' of $2 \times 2 \mathrm{~m}^{2}$ located $5 \mathrm{~m}$ from the ground at the middle point of the horizontal surface.

\begin{tabular}{|c|c|c|c|c|c|c|c|c|c|c|c|c|}
\hline \multicolumn{13}{|c|}{ Distance in the $X$-Axis to the Rectangle's Plane (m) } \\
\hline & & 0 & 1 & 2 & 3 & 4 & 5 & 6 & 7 & 8 & 9 & 10 \\
\hline & \multicolumn{12}{|c|}{ Dimensionless Configuration Factor $\mathbf{f}_{12}$ (i.e., Divided by $\pi$ ) } \\
\hline & 0 & 0.00000 & 0.00000 & 0.00000 & 0.00000 & 0.00000 & 0.00000 & 0.00000 & 0.00000 & 0.00000 & 0.00000 & 0.00000 \\
\hline & 1 & 0.00203 & 0.00279 & 0.00371 & 0.00469 & 0.00546 & 0.00577 & 0.00546 & 0.00469 & 0.00371 & 0.00279 & 0.00203 \\
\hline & 2 & 0.00368 & 0.00497 & 0.00650 & 0.00807 & 0.00930 & 0.00977 & 0.00930 & 0.00807 & 0.00650 & 0.00497 & 0.00368 \\
\hline Distance in the $Y$-axis & 3 & 0.00473 & 0.00624 & 0.00796 & 0.00966 & 0.01096 & 0.01144 & 0.01096 & 0.00966 & 0.00796 & 0.00624 & 0.00473 \\
\hline \multirow{7}{*}{ Rectangles Plane (m) } & 4 & 0.00519 & 0.00665 & 0.00825 & 0.00977 & 0.01090 & 0.0132 & 0.01090 & 0.00977 & 0.00825 & 0.00665 & 0.00519 \\
\hline & 5 & 0.00517 & 0.00644 & 0.00778 & 0.00901 & 0.00989 & 0.01021 & 0.00989 & 0.00901 & 0.00778 & 0.00644 & 0.00517 \\
\hline & 6 & 0.00486 & 0.00590 & 0.00695 & 0.00788 & 0.00852 & 0.00876 & 0.00852 & 0.00788 & 0.00695 & 0.00590 & 0.00486 \\
\hline & 7 & 0.00440 & 0.00521 & 0.00600 & 0.00668 & 0.00715 & 0.00732 & 0.00715 & 0.00668 & 0.00600 & 0.00521 & 0.00440 \\
\hline & 8 & 0.00389 & 0.00451 & 0.00510 & 0.00559 & 0.00592 & 0.00604 & 0.00592 & 0.00559 & 0.00510 & 0.00451 & 0.00389 \\
\hline & 9 & 0.00339 & 0.00385 & 0.00429 & 0.00464 & 0.00488 & 0.00496 & 0.00488 & 0.00464 & 0.00429 & 0.00385 & 0.00339 \\
\hline & 10 & 0.00293 & 0.00328 & 0.00360 & 0.00386 & 0.00402 & 0.00408 & 0.00402 & 0.00386 & 0.00360 & 0.00328 & 0.00293 \\
\hline
\end{tabular}

Table 2. Configuration factors of an area of $5 \times 10 \mathrm{~m}^{2}$ (the values that are omitted are obviously symmetrical) illuminated by a 'rectangle' of $2 \times 2 \mathrm{~m}^{2}$. Values obtained by analytical methods ([6], pp. 183-184).

\begin{tabular}{llcccccc}
\hline Distance in the $X$-Axis to the Rectangle's Plane (m) \\
\hline
\end{tabular}




\section{Discussion}

\subsection{Convergence}

To test the convergence of the method we need to gradually decrease the size of the elements and show how, by doing so, the form factors converge towards a certain value which must be increasingly closer to that calculated analytically.

One of the advantages of our algorithm is that by simply modifying the value of the sliders to increase the partitions we obtain the new results rapidly, and proof of convergence is readily available.

In the ensuing figure (Figure 14), we found the results by raising the number of partitions or finite elements from $10 \times 10=100$ elements to $100 \times 100=10,000$ elements, with a computing time of only 136 s (conventional computer).
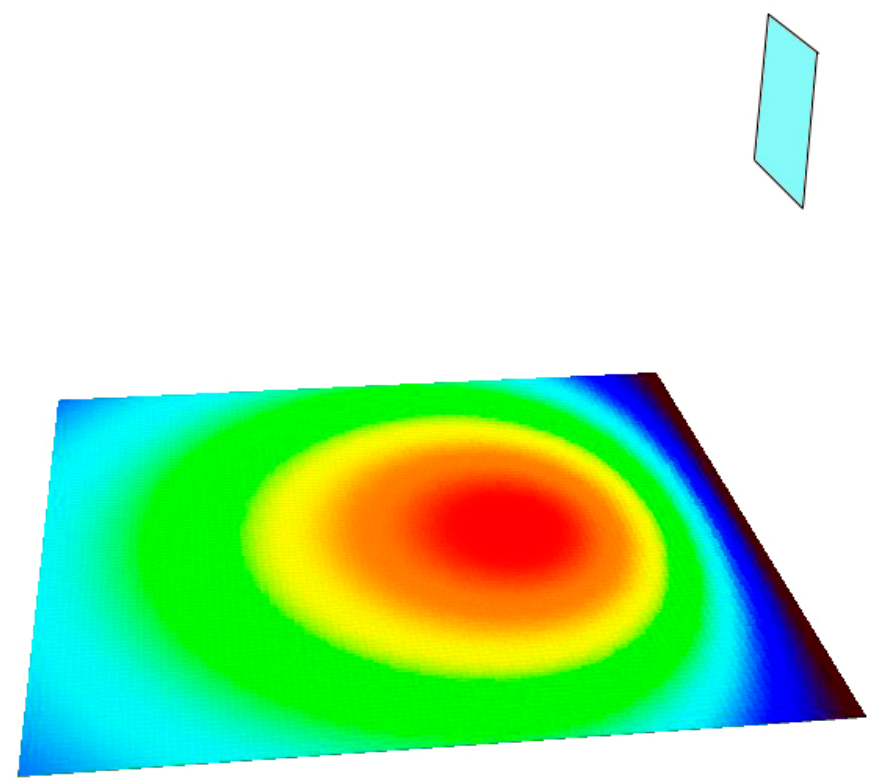

Figure 14. The color map of the configuration factor obtained by discretization for each $10 \mathrm{~cm}$ (10201 values) over an area of $10 \times 10 \mathrm{~m}^{2}$ radiated by a 'rectangle' of $2 \times 2 \mathrm{~m}^{2}, 5 \mathrm{~m}$ over the horizontal surface at its middle axis.

The following figure (Figure 15) shows the values obtained for the form factor by discretizing the surface of $10 \times 10 \mathrm{~m}$ in 121, 441, 961, 1681, 2601, 3721, 5041, 6561, 8281, 10,201, 12,321, 14,641, $17,161,19,881$ and 22,801 points, respectively (the value obtained for 22,801 points is $\mathrm{F}_{12}=0.006165497$ ), showing a clear convergence towards the value $\mathrm{F}_{12}=0.0062$ analytically calculated [6].

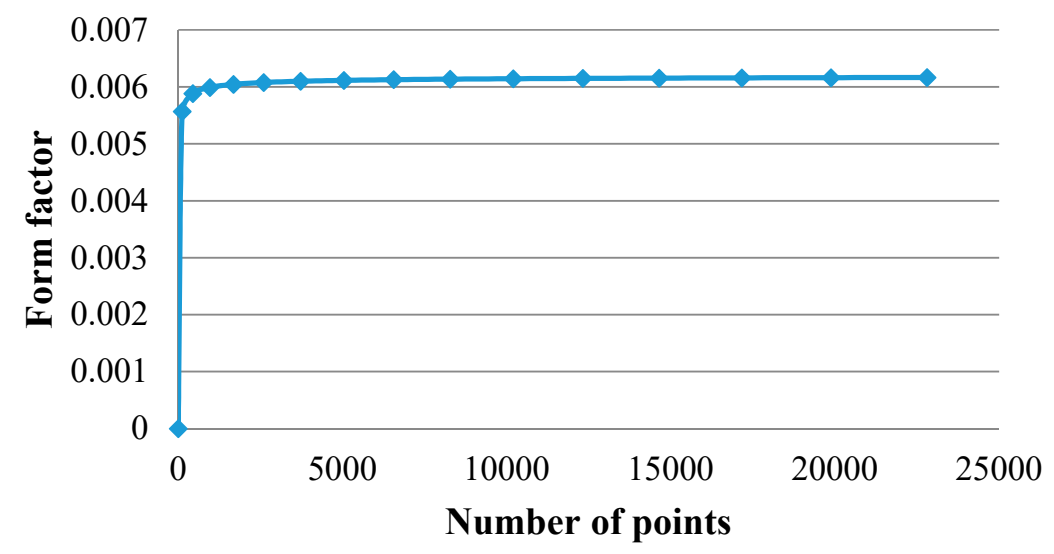

Figure 15. Convergence of the algorithm for the previously studied case. 


\subsection{Versatility of the Method}

As noted above, the difficulties in obtaining analytical solutions for emitters with forms other than the trivial are enormous, and even in many cases impossible. However, the method presented here allows solutions with high accuracy, limited only by the performance of the computer, for any type of emitters and irrespective of the orientation.

As a more complex example, we have calculated the configuration factors on a $10 \times 10 \mathrm{~m}$ horizontal surface, radiated by an elliptical emitter whose major and minor axes are $2 \mathrm{~m}$ and $1 \mathrm{~m}$ respectively and whose lowest point is located at a height of $5 \mathrm{~m}$. As before, vertical axis of the ellipse coincides with the middle point of the horizontal surface in $5 \mathrm{~m}$ (Figure 16). Notice that, for the time being, the elliptical shape cannot be solved analytically due the appearance of elliptic integrals in the process [9].

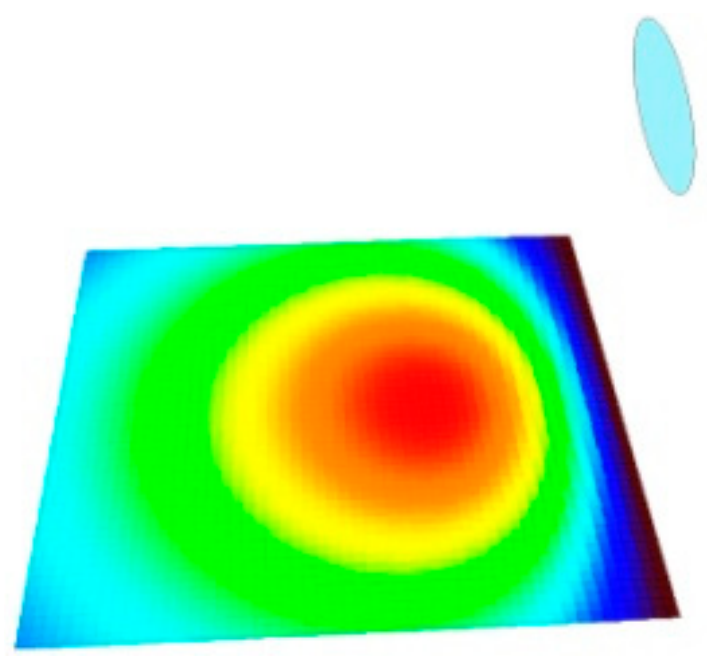

Figure 16. The color map of the configuration factor obtained by the discretization per each $20 \mathrm{~cm}$ (2601 values) of an area of $10 \times 10 \mathrm{~m}^{2}$ illuminated by an Ellipse of $2 \mathrm{~m}$ of major semi-axis and $1 \mathrm{~m}$ of minor semi-axis located $5 \mathrm{~m}$ from the ground in the middle point of the side of the horizontal surface.

Finally, we have simulated the configuration factors on a $10 \times 10 \mathrm{~m}$ horizontal surface, given by an arbitrary emitter in the shape of a scalene triangle of the dimensions depicted in Figure 17. The graphic results appear in Figure 18 and the numeric values in Table 3.

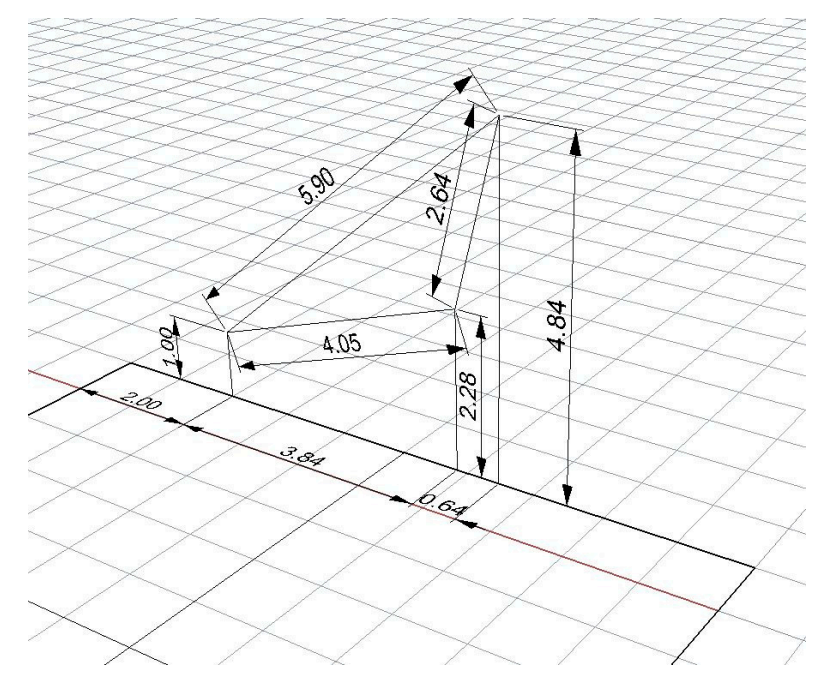

Figure 17. Sketch of the dimensions of a vertical scalene triangle radiating over a horizontal area of $10 \times 10 \mathrm{~m}^{2}$. 
Table 3. Configuration factors of an area of $10 \times 10 \mathrm{~m}^{2}$ illuminated by a 'scalene triangle'.

\begin{tabular}{|c|c|c|c|c|c|c|c|c|c|c|c|c|}
\hline \multicolumn{13}{|c|}{ Distance in the $X$-Axis to the Triangle's Plane (m) } \\
\hline & & $\mathbf{0}$ & 1 & 2 & 3 & 4 & 5 & 6 & 7 & 8 & 9 & 10 \\
\hline & \multicolumn{12}{|c|}{ Dimensionless Configuration Factor $\mathrm{f}_{12}$ (i.e., Divided by $\pi$ ) } \\
\hline & 0 & 0.00000 & 0.00000 & 0.00000 & 0.00000 & 0.00000 & 0.00000 & 0.00000 & 0.00000 & 0.00000 & 0.00000 & 0.00000 \\
\hline & 1 & 0.00329 & 0.00593 & 0.01103 & 0.02039 & 0.03471 & 0.04974 & 0.05761 & 0.05139 & 0.03030 & 0.01256 & 0.005371 \\
\hline Distance in the $Y$-axis to the & 2 & 0.00554 & 0.00942 & 0.01614 & 0.02677 & 0.04031 & 0.05151 & 0.05363 & 0.04433 & 0.02858 & 0.01539 & 0.00799 \\
\hline \multirow[t]{8}{*}{ Triangles Plane (m) } & 3 & 0.00642 & 0.01014 & 0.01581 & 0.02349 & 0.03169 & 0.03715 & 0.03682 & 0.03064 & 0.02171 & 0.01371 & 0.00825 \\
\hline & 4 & 0.00627 & 0.00921 & 0.01318 & 0.01789 & 0.02229 & 0.02476 & 0.02415 & 0.02067 & 0.01578 & 0.01106 & 0.00740 \\
\hline & 5 & 0.00559 & 0.00769 & 0.01027 & 0.01303 & 0.01535 & 0.01650 & 0.01604 & 0.01413 & 0.01141 & 0.00862 & 0.00623 \\
\hline & 6 & 0.00474 & 0.00619 & 0.00782 & 0.00943 & 0.01069 & 0.01126 & 0.01097 & 0.00989 & 0.00833 & 0.00664 & 0.00509 \\
\hline & 7 & 0.00392 & 0.00490 & 0.00593 & 0.00689 & 0.00761 & 0.00791 & 0.00773 & 0.00710 & 0.00618 & 0.00513 & 0.00411 \\
\hline & 8 & 0.00321 & 0.00387 & 0.00453 & 0.00513 & 0.00554 & 0.00572 & 0.00560 & 0.00552 & 0.00465 & 0.00399 & 0.00332 \\
\hline & 9 & 0.00262 & 0.00307 & 0.00351 & 0.00388 & 0.00414 & 0.00424 & 0.00416 & 0.00393 & 0.00357 & 0.00314 & 0.00268 \\
\hline & 10 & 0.00214 & 0.00246 & 0.00275 & 0.00299 & 0.00315 & 0.00322 & 0.00316 & 0.00302 & 0.00278 & 0.00249 & 0.00218 \\
\hline
\end{tabular}

Notice that, in the case of the ellipse and the scalene triangle unlike the rectangle, it is not possible nowadays to calculate the configuration factors by any other procedure. 


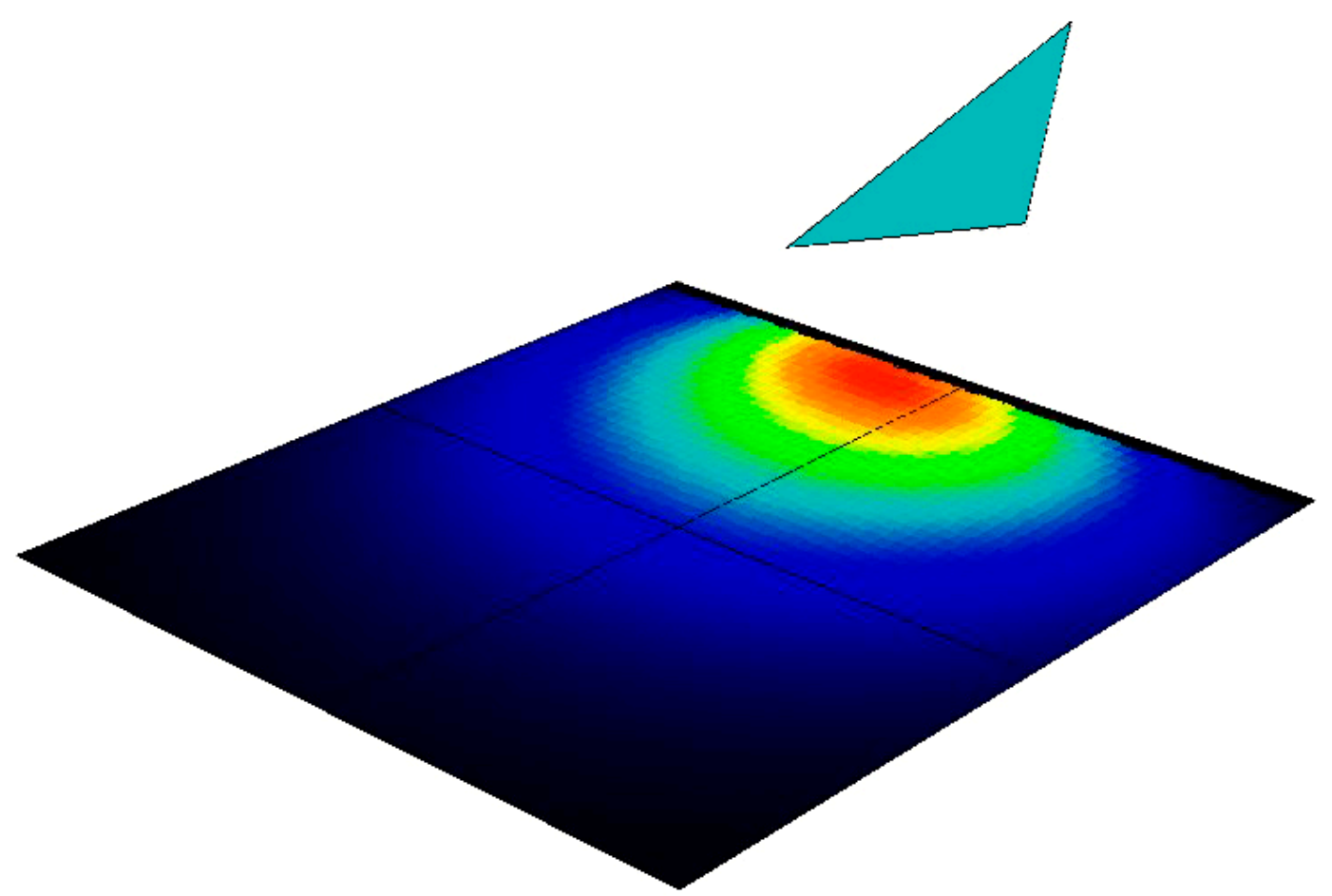

Figure 18. Map of the configuration factors obtained by the discretization per each $20 \mathrm{~cm}$ (2601 values) of an area of $10 \times 10 \mathrm{~m}^{2}$ illuminated by an arbitrary scalene triangle with dimensions expressed in Figure 17.

\section{Conclusions and Future Aims}

Given the accurate convergence of the values obtained for the particular case of rectangular emitters and receivers within a cuboid, we consider that the graphical interface program that we have proposed is fully accomplished. However, for the time being, we have to warn that there is no feasible way to validate the other figures presented, such as ellipse and scalene triangle.

Nevertheless, we believe that a complex physical-mathematical problem, which has occupied the mind of scholars for decades if not centuries, is solved with perfect ease in a semi-automatic manner.

Ensuing research will extend the procedure to other types of three-dimensional spaces where analytical calculations were proposed but still remain underdeveloped, for instance curvilinear sources in non-cuboidal domains affected by interferences and obstacles [9].

A much wider repertoire of possible radiative exchanges would be achieved in this fashion, with relative simplicity and accuracy and without alternative. The repercussions of such findings would be wide-ranging in fields as diverse as aerospace technologies [10], Light Emitting Diodes (LED), radiotherapy medicine, risk prevention, acoustics and architecture [11]. On an industrial basis, we consider that the vast field of artificial lighting and design of luminaries will immediately benefit from our methods.

Whereas the findings hereby exposed would represent a major achievement in Optics, precisely for the realms of Heat Transfer and Lighting, it is understood that our contribution has been timely aided by the enormous potential of Algorithms Aided Design in the numerical resolution of physical-mathematical problems that, when approached directly, are extremely difficult to accomplish (if not altogether impossible).

Author Contributions: Conceptualization, J.-M.C.-L.; data curation, F.S.-A.; software, F.S.-A.; investigation, J.-M.C.-L.; writing-original draft, F.S.-A. and J.-M.C.-L. All authors have read and agreed to the published version of the manuscript.

Funding: This research received no external funding. 
Acknowledgments: The authors would like to warmly thank Enrique Salguero-Jiménez for his unselfish collaboration in the preparation of most of the figures that appear in this work. Cabeza-Lainez wants to honor with the article the figure of the late Engineer Jose Ramon Perez de Lama. Our piece of writing has been composed in praise of Tomomi Odajima San.

Conflicts of Interest: The authors declare none.

\section{Nomenclature}

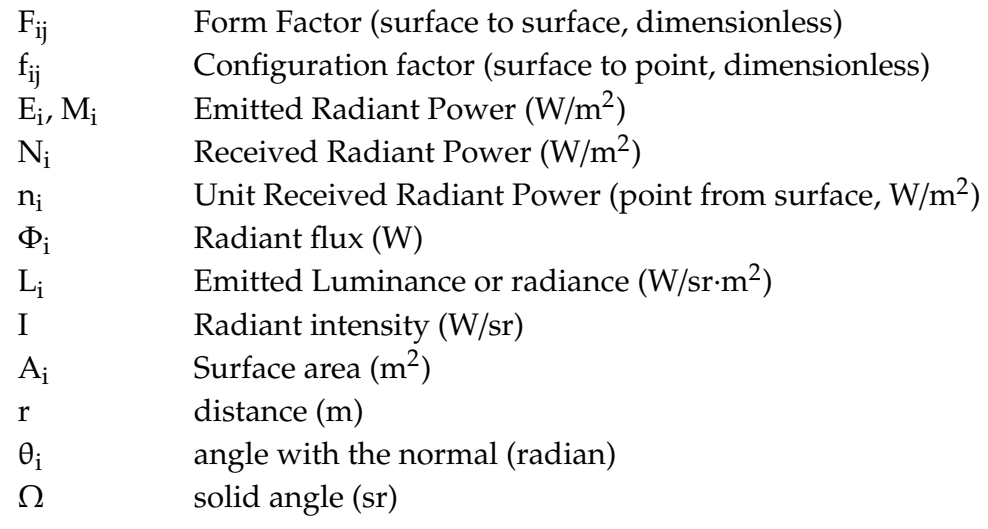

\section{Appendix A. Further Details on the Calculations of Basic Configuration Factors}

\section{Appendix A.1. Triangles and Shapes Composed of Triangles}

In the first case, which is also the most elementary, we would substitute the value of the cosine as expressed in Equation (16) and find the fundamental double-integral equation,

$$
E=L \int_{0}^{b} \int_{0}^{z} \frac{y^{2}}{\left(x^{2}+y^{2}+z^{2}\right)^{2}} d z d x
$$

Note that we write again $\mathrm{E}$ (surface-to-point received power) instead of the previous magnitude $\mathrm{n}$ because this nomenclature is more usual. We have chosen an instant of time $t$ in which the luminance or radiance $\mathrm{L}$ can be considered constant, keeping in mind that $\mathrm{L} \pi=\mathrm{M}$.

We will solve it as an example of the general ensuing procedure to attest to its difficulty.

The upper line of triangle $A_{1}$ is defined by equation $\mathrm{z}=\mathrm{x}(\mathrm{a} / \mathrm{b})$, and therefore, replacing the new value of $\mathrm{z}$ :

$$
E=L \int_{0}^{b} \int_{0}^{\frac{a}{b} x} \frac{y^{2}}{\left(x^{2}+y^{2}+z^{2}\right)^{2}} d z d x
$$

Solving the integral of the second member of Equation (A2), (the details of the resolution of this and all other integrals presented in this article can be consulted in [6]) we obtain:

$$
E=\frac{L}{2}\left[\frac{b}{\sqrt{y^{2}+b^{2}}} \tan ^{-1} \frac{a}{\sqrt{y^{2}+b^{2}}}\right]
$$

Respectively, the upper triangle, surface $\mathrm{A}_{2}$ in Figure 2, would have yielded:

$$
E=\frac{L}{2}\left[\frac{a}{\sqrt{y^{2}+b^{2}}} \tan ^{-1} \frac{b}{\sqrt{y^{2}+b^{2}}}\right]
$$




\section{Appendix A.2. Rectangle}

The full rectangle is the sum of Equations (A3) and (A4), that is:

$$
\mathrm{E}=\frac{\mathrm{L}}{2}\left[\frac{\mathrm{a}}{\sqrt{\mathrm{y}^{2}+\mathrm{a}^{2}}} \tan ^{-1} \frac{\mathrm{b}}{\sqrt{\mathrm{y}^{2}+\mathrm{a}^{2}}}+\frac{\mathrm{b}}{\sqrt{\mathrm{y}^{2}+\mathrm{b}^{2}}} \tan ^{-1} \frac{\mathrm{a}}{\sqrt{\mathrm{y}^{2}+\mathrm{b}^{2}}}\right]
$$

We would ask the reader to understand that, with all this complexity, we have only obtained the value of radiative exchange at a single point located precisely under the vertex of the rectangle and in a particular direction at right angles to the surface source.

\section{Appendix A.3. Square}

For example, in a square whose side is $d=2 a=2 b$; the final expression is the result of multiplying by eight the basic triangle previously obtained, which yields:

$$
\mathrm{E}=4 \mathrm{~L}\left[\frac{\mathrm{d}}{\sqrt{4 \mathrm{y}^{2}+\mathrm{d}^{2}}} \tan ^{-1} \frac{\mathrm{d}}{\sqrt{4 \mathrm{y}^{2}+\mathrm{d}^{2}}}\right]
$$

\section{Appendix A.4. Equilateral Triangle}

If we contemplate the figure of the equilateral triangle (Figure A1), the expression will now be six times the rotated triangle, and we receive:

$$
E=3 L\left[\frac{d}{\sqrt{12 y^{2}+d^{2}}} \tan ^{-1} \frac{d \sqrt{3}}{\sqrt{12 y^{2}+d^{2}}}\right]
$$

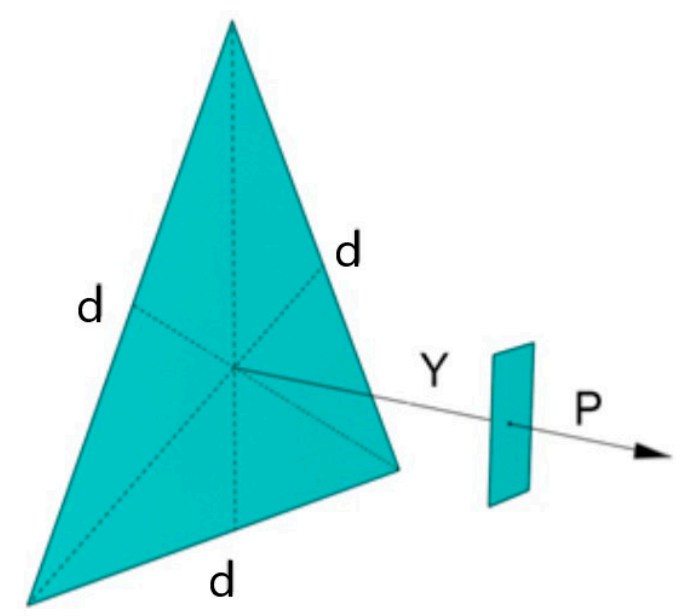

Figure A1. Configuration factor between an equilateral triangle composed of six rotated triangles and a point in a parallel plane.

\section{Appendix A.5. Hexagon}

In the specific case of the hexagon (Figure A2), we would need 12 times the rotated triangle, which, expressed as a function of the side $d$, gives us:

$$
E=6 L\left[\frac{d \sqrt{3}}{\sqrt{4 y^{2}+3 d^{2}}} \tan ^{-1} \frac{d}{\sqrt{4 y^{2}+3 d^{2}}}\right]
$$




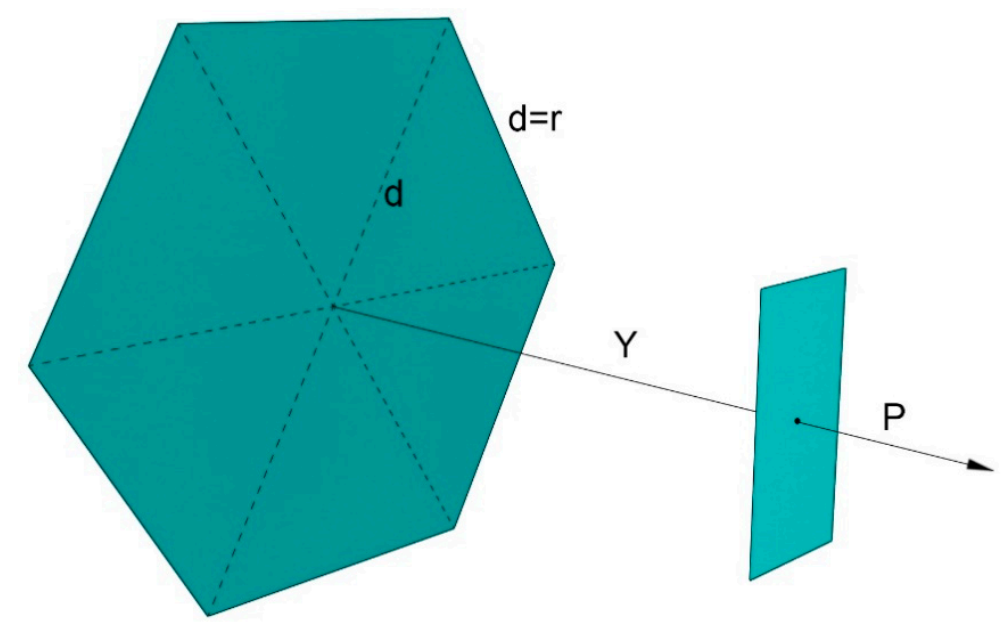

Figure A2. Configuration factor between a hexagon composed of 12 rotated triangles and a point under the center in a parallel plane.

\section{Appendix A.6. Circle}

Finally, the limiting case of all simple figures composed of triangles is a regular $\mathrm{n} \rightarrow \infty$ sided polygon (Figure A3) whose limit is obviously a circle and in it:

$$
E=\frac{L}{2} . \lim _{a \rightarrow 0 ; n \rightarrow \infty}\left\{n \cdot\left[\frac{b}{\sqrt{y^{2}+b^{2}}} \tan ^{-1} \frac{a}{\sqrt{y^{2}+b^{2}}}\right]\right\}
$$

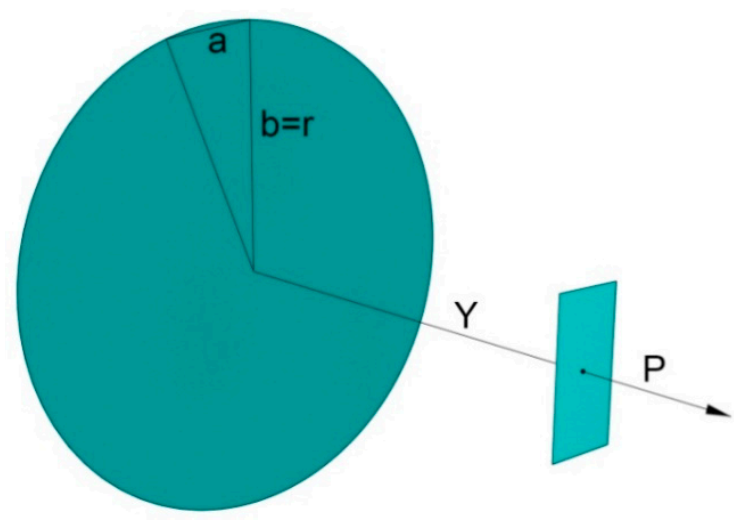

Figure A3. Configuration factor between a circle consisting of $\mathrm{n}$ rotated triangles and a point from the normal to the center that is located in a plane parallel to the circle.

Since $a \rightarrow 0$ we can substitute the angle (arc of tangent) for its tangent, finding:

$$
E=\frac{L}{2} \lim _{n \rightarrow \infty} n\left[\frac{b a}{y^{2}+b^{2}}\right]
$$

but when $n \rightarrow \infty$, the product $n \cdot a$ is equivalent to $2 \pi b$ or $2 \pi r$ (the length of the circumference) and, therefore, in a more usual formulation the amount of energy as a function of radius and distance is found [7]:

$$
E=L \pi\left[\frac{b^{2}}{b^{2}+y^{2}}\right]=L \pi\left[\frac{r^{2}}{y^{2}+r^{2}}\right]
$$




\section{Appendix A.7. Calculations in a Plane Perpendicular to the Figure}

Imploring the patience of the reader, we need to remark that in the previous paragraphs we have limited ourselves to establishing the component of radiation that was perpendicular to the emitting surface considered (the one that is received onto a parallel plane), at a particular point under the vertex or the center of the figure.

Imagine if you can the complexity that arises when trying to find the solution for points moving freely in space, which is almost unfathomable.

Even so, as is logical, radiance should be treated like a vector, thus, if we want to complete the problem we will have to also find the values of the two components that are missing and remain parallel to the area considered. The defining integral then turns out to be (if we multiply by the corresponding cosine):

$$
E=L \iint \frac{z y}{\left(x^{2}+y^{2}+z^{2}\right)} d x d z
$$

Applying this proposition to the first case considered we would find that if $\mathrm{Y}$ (the distance) is a constant, the first part of the integral becomes not lengthy and the equation adopts the value:

$$
E=\frac{L}{2}\left[\tan ^{-1} \frac{b}{y}-\frac{b}{\sqrt{a^{2}+b^{2}}} \tan ^{-1} \frac{\sqrt{a^{2}+b^{2}}}{y}\right]
$$

For the entire rectangle, the boundaries would lie between a and 0 , instead of using a function of $\mathrm{x}$. Grouping the sum of the two terms, it turns out that:

$$
E=\frac{L}{2}\left[\tan ^{-1} \frac{b}{y}-\frac{y}{\sqrt{a^{2}+y^{2}}} \tan ^{-1} \frac{b}{\sqrt{a^{2}+y^{2}}}\right]
$$

To find the amount of the term due to the upper triangle in Figure 3, we would need to change the integration limits (from $\mathrm{z}=\mathrm{a}$ to $\mathrm{z}=\mathrm{ax} / \mathrm{b}$ ) and consequently:

$$
E=\frac{L}{2}\left[\frac{b}{\sqrt{a^{2}+b^{2}}} \tan ^{-1} \frac{\sqrt{a^{2}+b^{2}}}{y}-\frac{y}{\sqrt{a^{2}+y^{2}}} \tan ^{-1} \frac{b}{\sqrt{a^{2}+y^{2}}}\right]
$$

which could have been deduced by logic by subtracting Equation (A13) from Equation (A14).

Adding and subtracting rectangles would allow us to obtain the result for a rectangular shape over an unlimited horizontal surface as described in Figure 4 and Equation (28). Only a few months ago Cabeza-Lainez obtained a similar solution for a triangle with a horizontal side and also for circular sectors if the center of the vertical circle lies on the horizontal surface. [11]

In this quite elaborate fashion, we have discovered how complex it becomes to find an analytical expression to solve the problem if we only alter the coordinate plane of calculation not to mention figures of geometry other than the regular triangle or rectangle. That is why we concluded that a graphical solution based on parametric design would imply a much more general solution and extraordinarily simplify the issue. This is the reasoning behind our method.

\section{Appendix B. Particulars of the Numerical Validation of the Projected Solid-Angle Principle}

\section{Numerical Validation for a Rectangle}

Suppose that the emitter is a rectangle $1 \mathrm{~m}$. high and $3 \mathrm{~m}$. wide and we try to calculate the configuration factor at a point $P$ that lies in the plane of symmetry of the rectangle at a longitudinal distance of $2 \mathrm{~m}$ and is located $1 \mathrm{~m}$ below the bottom edge of the said rectangle (Figure A4).

So, $\mathrm{D}=2, \mathrm{H}_{0}=2, \mathrm{H}_{1}=1$ and $\mathrm{M}=1.5$.

With this data, Equation (28) yields the well-known result:

$$
\mathrm{n}=\mathrm{L}\left[\frac{2}{\sqrt{5}} \tan ^{-1} \frac{1.5}{\sqrt{5}}-\frac{2}{\sqrt{8}} \tan ^{-1} \frac{1.5}{\sqrt{8}}\right]=0.18368 \mathrm{~L}\left(\mathrm{~W} / \mathrm{m}^{2}\right)
$$

However, if we try to check this value by means of the projected solid-angle law, it becomes extremely difficult, as we shall see. 


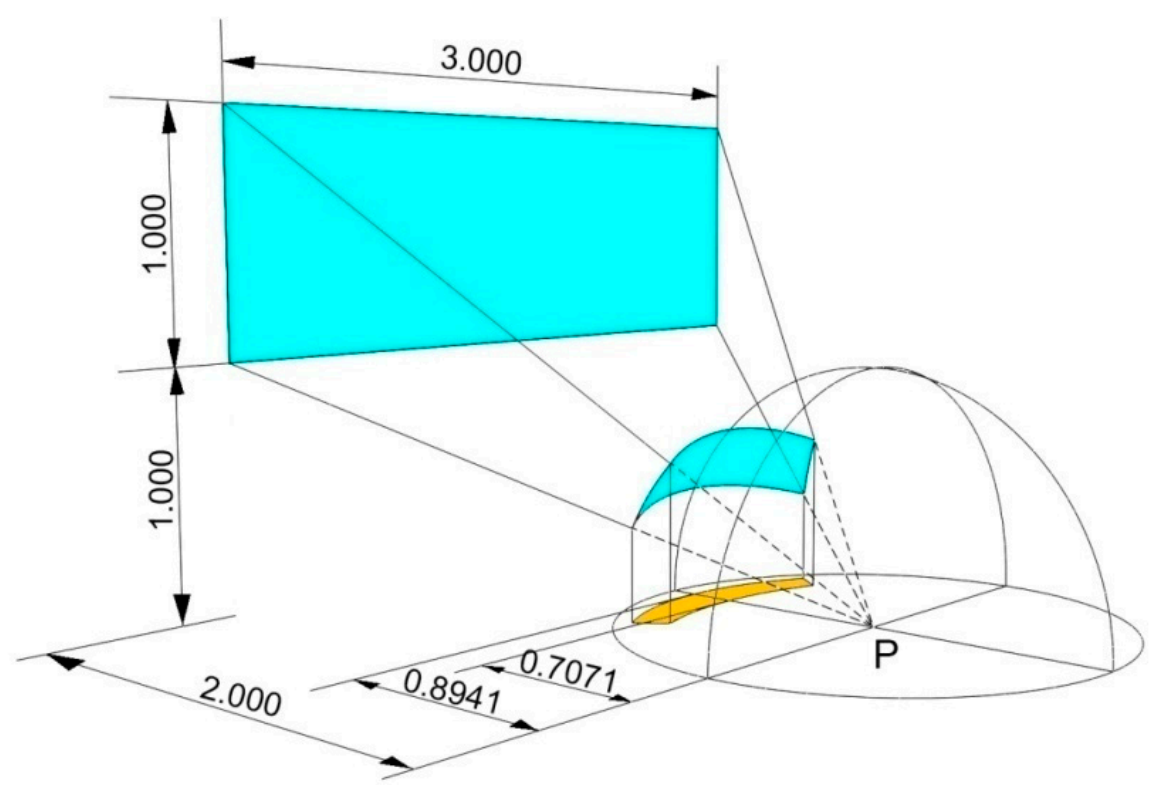

Figure A4. Scheme of a 'rectangle' type to employ in calculations.

Since $\mathrm{f}_{12}$ must be a dimensionless configuration factor, we ought to divide by the area of the projected unit sphere, that is $\pi r^{2}=\pi$, from which we would arrive at:

$$
\mathrm{f}_{12}=\mathrm{n} /(\mathrm{L} \pi)=0.0584
$$

We can geometrically obtain the lengths of the minor semi-axes of the projection of the two ellipses involved which are, respectively, $b_{1}=0.8941$ and $b_{0}=0.7071$. The major semi-axis is logically $1 \mathrm{~m}$.

Strangely, the only way to solve the problem is by changing the coordinates to (very rare) generalized polar coordinates, and thus we would have:

$$
\mathrm{x}=\mathrm{a} \rho \cos \theta ; \mathrm{y}=\mathrm{b} \rho \cos \theta,
$$

thus, the differential element yields $\mathrm{dA}=\mathrm{a} b \rho \mathrm{d} \rho \mathrm{d} \theta$.

This is, in fact, the only possible procedure to obtain the area of an elliptic sector:

$$
A=\int_{\theta_{2}}^{\theta_{1}} \int_{0}^{1} \mathrm{ab} \rho \mathrm{d} \rho \mathrm{d} \theta=\mathrm{ab} \int_{\theta_{2}}^{\theta_{1}}\left[\frac{\rho^{2}}{2}\right]_{0}^{1} \mathrm{~d} \theta=\mathrm{ab} \int_{\theta_{2}}^{\theta_{1}} \frac{1}{2} \mathrm{~d} \theta=\mathrm{ab} \frac{\left(\theta_{1}-\theta_{2}\right)}{2}
$$

Now it will suffice to find the values of $\theta_{1}$ and $\theta_{2}$ under the new coordinate system. Since the angles are equal with respect to the axis of symmetry of the 'rectangle', the previous expression can be transformed into the simpler product: $\mathrm{a} \cdot \mathrm{b} \cdot \theta_{\mathrm{i}}$.

For the larger ellipse $\mathrm{y} / \mathrm{x}=2 / 1.5$ (by proportion); $\mathrm{a}=1, \mathrm{~b}=0.8944$.

Then $\theta_{1}=\tan ^{-1}(2 /(1.5 \cdot 0.8944))$, which is 0.9799 ; and the complementary with respect to $\pi / 2$ is 0.5908 pseudo-radians (a new local unit only valid for 'each' ellipse).

The area of the larger ellipse is $0.5984 \cdot 0.8944=0.5284$ and accordingly for the small ellipse the area would be 0.3447 ; therefore, the final area yields $0.5284-0.3447=0.1837$.

This quantity divided by $\pi$ provides

$$
\mathrm{f}_{12}=0.0584 \text {. }
$$

'Quod erat demonstrandum'.

It is important to stress that the values obtained for the configuration factors through the application of the principle of the projected solid angle are not approximations to those obtained by analytical calculations but are identical. Such a demonstration, simple as it is, due to the extreme difficulties arising with the integration of elliptic geometries, is a finding solely attributed to Cabeza-Lainez. In this way he has dissipated the clouds that once spread over the validity of such a convenient principle, for our method and beyond.

\section{Appendix C. The Recurrent Problem of Circular Emitters}

Circular emitters can be treated in the same way as any type of emitter by virtue of the law of the projected solid angle (Figure A5). Nonetheless, explicit solutions were unassailable [7,9]. 


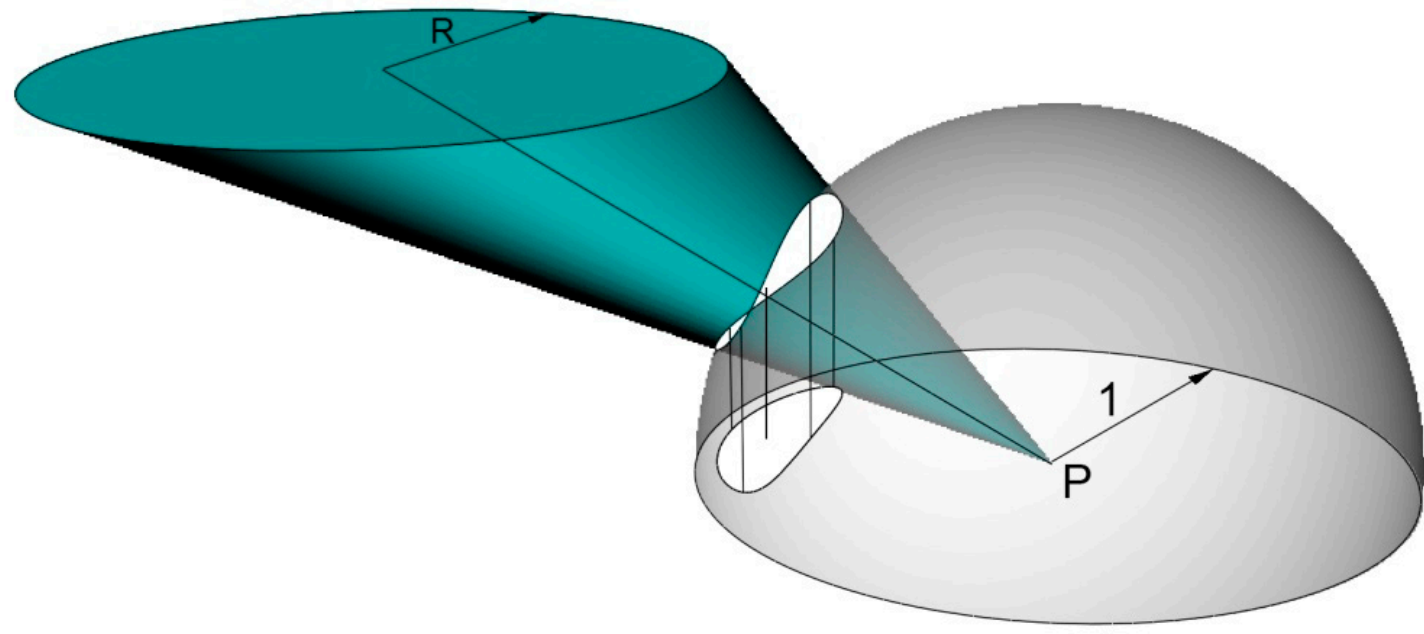

Figure A5. Projection on the horizontal plane of the intersection of an oblique cone and a unit radius sphere.

Cabeza-Lainez proposed that, through a so-called "pair of dejection" (Figure A6), we could determine the parameters of the cone that subtends the emitting circle of radius $R$, at point $P$.

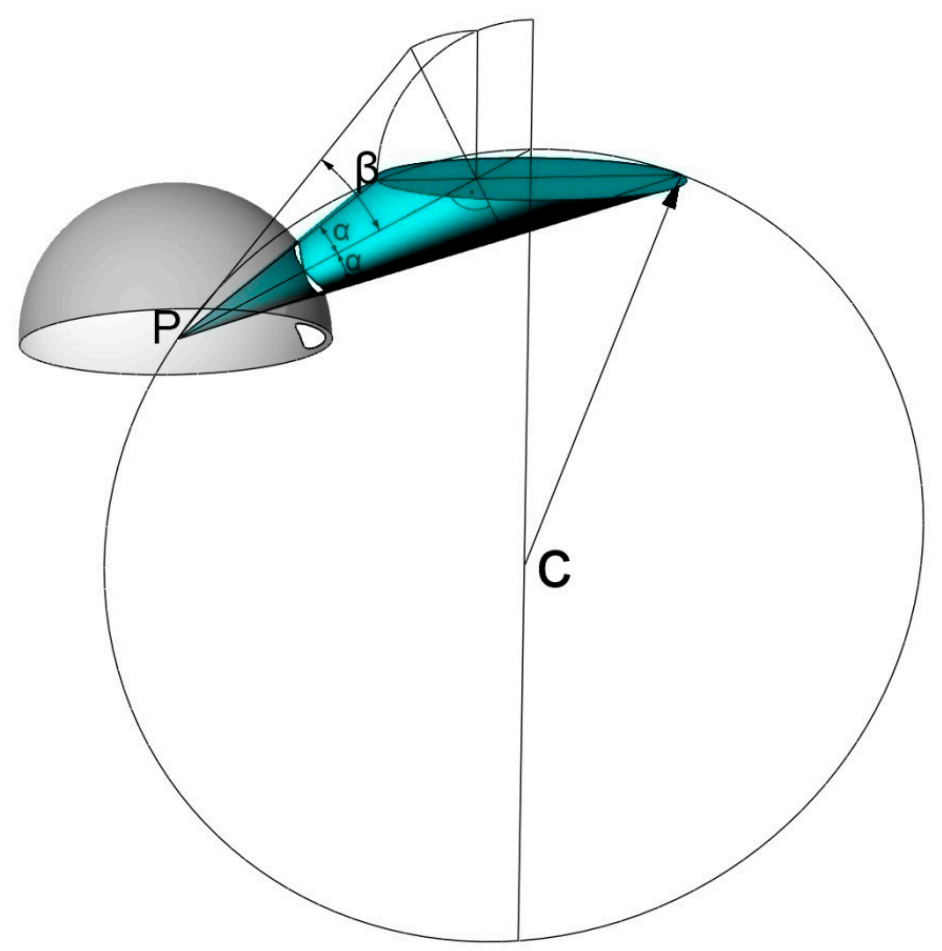

Figure A6. Procedure to find the main angles of the radiant cone.

The resulting equation of the elliptical cone is:

$$
\frac{x^{2}}{\sin ^{2} \alpha}+\frac{y^{2}}{\cos ^{2} \alpha \tan ^{2} \beta}=\frac{z^{2}}{\cos ^{2} \beta}
$$

The intersection between the oblique cone of parameters $\alpha$ and $\beta$ and a sphere of unit radius defines itself by the expression:

$$
x^{2}+y^{2}+z^{2}=1 \frac{x^{2}}{\sin ^{2} \alpha}+\frac{y^{2}}{\cos ^{2} \alpha \tan ^{2} \beta}=\frac{z^{2}}{\cos ^{2} \beta}
$$


Thus, we arrive at the miraculous equation of the intersection between the former cone and a sphere of unit radius with its center in the vertex of the cone (Equation (A20)); this curve that we have found is called Tomomi by Cabeza-Lainez [10] and yields the following parametric equations for its coordinates (Figure A7),

$$
x=\sin \alpha \cos t \cdot y=\sin \beta \sin t z=\sqrt{\cos ^{2} \alpha \cos ^{2} t+\cos ^{2} \beta \sin ^{2} t}
$$

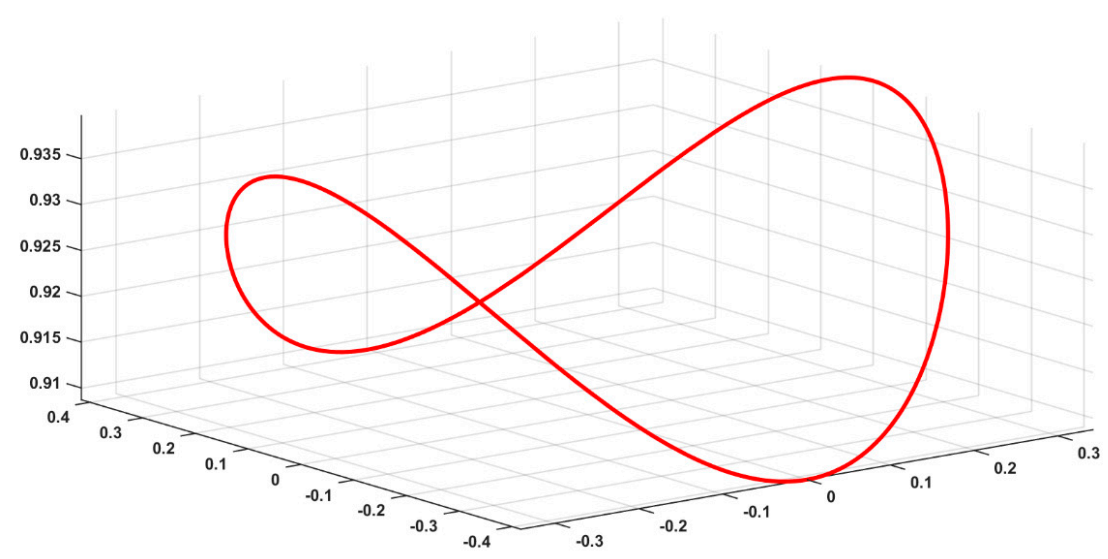

Figure A7. Axonometric view of Tomomi curve.

Looking for the planes of common symmetry between the sphere and the elliptical cone, and applying elementary theorems of intersections of quadrics, we can observe the Tomomi curve of Cabeza-Lainez as a branch of hyperbola or as an ellipse (Figure A8).

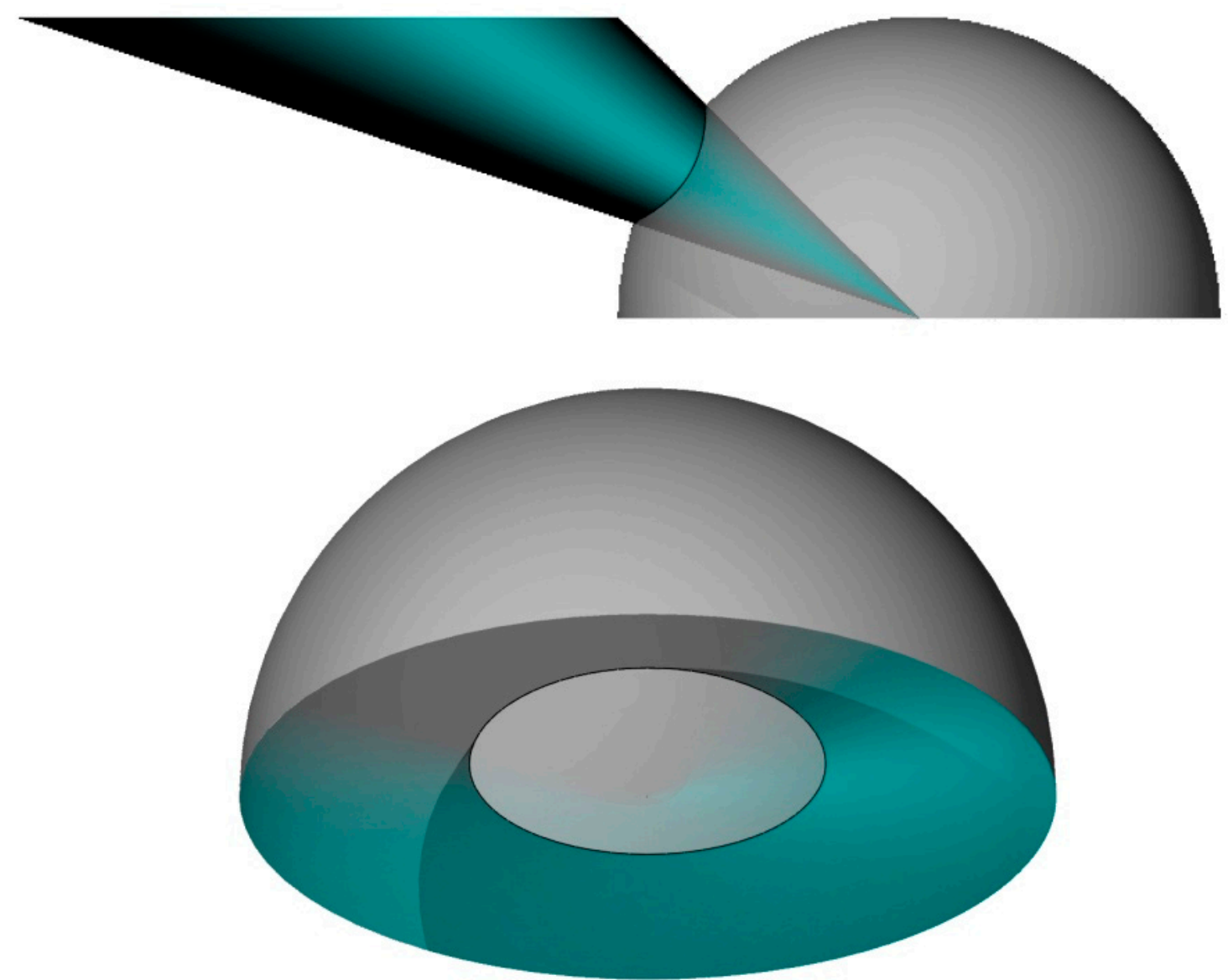

Figure A8. (Top). Projection of the intersection parallel to the axis of the cone: Hyperbola. (Bottom). Projection of the intersection normal to the axis of the cone: Ellipse. 
Although it is actually a fourth degree curve as shown (Figure A9) in the ensuing representation, even so it is not possible in most cases to find its enclosed area by numerical procedures [12].

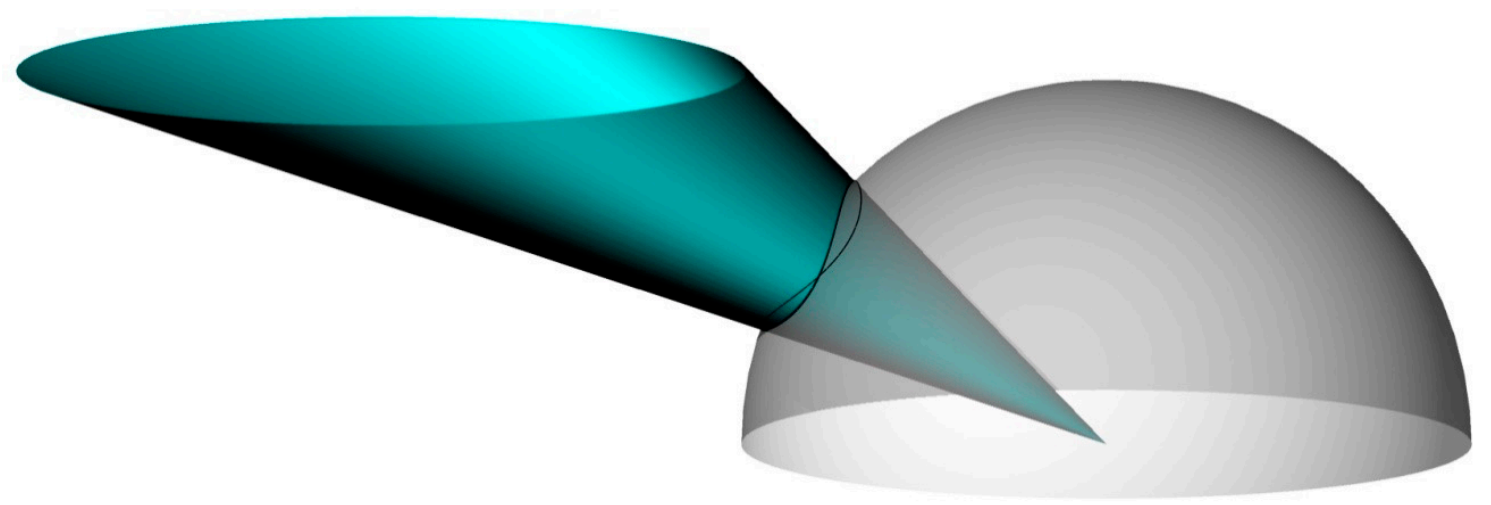

Figure A9. Perspective view of the Tomomi's curve.

\section{References}

1. Yamauchi, J. The amount of flux incident to rectangular floor through rectangular windows. Res. Electrotech. Lab. 1929, 250.

2. Moon, P.H. The Scientific Basis of Illumination Engineering; Dover Publications: New York, NY, USA, 2008.

3. Moon, P.H.; Spencer, D.E. The Photic Field; The MIT Press: Cambrige, MA, USA, 1981.

4. Lambert, J.H. Photometry, or, on the Measure and Gradations of Light, Colors, and Shade: Translation from the Latin of Photometria, Sive, De Mensura et Gradibus Luminis, Colorum et Umbrae, with Introductory Monograph and Notes by David L. DiLaura; Illuminating Engineering Society of North America: New York, NY, USA, 2001.

5. Cabeza-Lainez, J.M. Radiative performance of louvers. Simulation and examples in Asian Architecture. In Proceedings of the 6th International Conference on Indoor Air Quality, Ventilation \& Energy Conservation in Buildings (IAQVEC), Sendai, Japan, 28-31 October 2007; Volume 3, ISBN 978-4-86163-072-9 C3025\4762E.

6. Cabeza-Lainez, J.M. Fundamentos de Transferencia Radiante Luminosa o la Verdadera Naturaleza del Factor de Forma y sus Modelos de Cálculo; Netbiblio: Seville, Spain, 2010.

7. Cabeza-Lainez, J.M.; Pulido-Arcas, J.A. New configuration factors for curved surfaces. J. Quant. Spectrosc. Radiat. Transf. 2013, 117, 71-80. [CrossRef]

8. Tedeschi, A. AAD_Algorithms-Aided Design. Parametric Strategies Using Grasshopper ${ }^{\circledR}$; Le Penseur Publisher: Potenza, Italy, 2014; pp. 22-23.

9. Fock, V.A. Zur Berechnung der Beleuchtungsstärke. Z. Phys. 1924, 28, 102-113. [CrossRef]

10. Sasaki, K.; Sznajder, M. Analytical view factor solutions of a spherical cap from an infinitesimal surface. Int. J. Heat Mass Transf. 2020, 163, 120477. [CrossRef]

11. Salguero Andujar, F.; Rodriguez-Cunill, I.; Cabeza-Lainez, J.M. The problem of lighting in underground domes, vaults, and tunnel-like structures of antiquity; an application to the sustainability of prominent Asian heritage (India, Korea, China). Sustainability 2019, 11, 5865. [CrossRef]

12. Gershun, A. The Light Field (translated by P. Moon and G. Timoshenko). J. Math. Phys. 1939, 18, 51-151. [CrossRef]

Publisher's Note: MDPI stays neutral with regard to jurisdictional claims in published maps and institutional affiliations.

(C) 2020 by the authors. Licensee MDPI, Basel, Switzerland. This article is an open access article distributed under the terms and conditions of the Creative Commons Attribution (CC BY) license (http://creativecommons.org/licenses/by/4.0/). 